\title{
Lipidomic and transcriptomic analysis reveals reallocation of carbon flux from cuticular wax into plastid membrane lipids in a glossy "Newhall" navel orange mutant
}

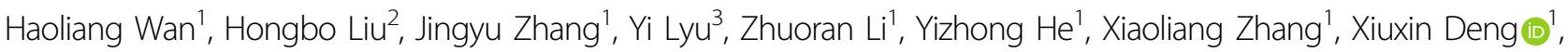 \\ Yariv Brotman ${ }^{4}$, Alisdair R. Fernie $\mathbb{B}^{5}$, Yunjiang Cheng $^{1}$ and Weiwei Wen $\mathbb{B}^{1}$
}

\begin{abstract}
Both cuticle and membrane lipids play essential roles in quality maintenance and disease resistance in fresh fruits. Many reports have indicated the modification of alternative branch pathways in epicuticular wax mutants; however, the specific alterations concerning lipids have not been clarified thus far. Here, we conducted a comprehensive, timeresolved lipidomic, and transcriptomic analysis on the "Newhall" navel orange (WT) and its glossy mutant (MT) "Gannan No. 1". The results revealed severely suppressed wax formation accompanied by significantly elevated production of 36-carbon plastid lipids with increasing fruit maturation in MT. Transcriptomics analysis further identified a series of key functional enzymes and transcription factors putatively involved in the biosynthesis pathways of wax and membrane lipids. Moreover, the high accumulation of jasmonic acid (JA) in MT was possibly due to the need to maintain plastid lipid homeostasis, as the expression levels of two significantly upregulated lipases (CsDAD1 and CsDALL2) were positively correlated with plastid lipids and characterized to hydrolyze plastid lipids to increase the JA content. Our results will provide new insights into the molecular mechanisms underlying the natural variation of plant lipids to lay a foundation for the quality improvement of citrus fruit.
\end{abstract}

\section{Introduction}

Waxing and polishing are key steps in the commercialization of fresh citrus. However, due to its unique chemical properties, the synthetic wax used for this purpose blocks stomatal pores and affects fruit quality. Therefore, the cultivation of fruits with desirable colors, high glossiness, fine internal quality, and strong antibacterial properties is of considerable importance. As the first external barrier of plants that come into contact with the

\footnotetext{
Correspondence: Yunjiang Cheng (yjcheng@mail.hzau.edu.cn) or Weiwei Wen (wwwen@mail.hzau.edu.cn)

${ }^{1}$ Key Laboratory of Horticultural Plant Biology (MOE), College of Horticulture and Forestry Sciences, Huazhong Agricultural University, 430070 Wuhan, China ${ }^{2}$ National Key Laboratory of Crop Genetic Improvement, Huazhong

Agricultural University, 430070 Wuhan, China

Full list of author information is available at the end of the article.

These authors contributed equally: Haoliang Wan, Hongbo Liu
}

environment, epicuticular wax has been reported to play important roles in water retention, gas exchange $\mathrm{e}^{1,2}$, and surface glossiness of citrus fruits ${ }^{3,4}$.

In conjunction with the external protection of the cuticle, membrane lipids directly protect plant cells and organelles ${ }^{5}$, and plastid membrane lipids are the principal constituents of plant membrane lipid systems. Unlike phosphatidylcholine (PC) and phosphatidylethanolamine (PE), which are dominant in the plasma membrane, galactoglycerolipids, such as monogalactosyldiacylglycerols (MGDGs) and digalactosyldiacylglycerols (DGDGs), together with a fraction $(\sim 10 \%)$ of phosphatidylglycerols (PGs), serve as the structural lipids that sustain the delicate skeleton of the plastid membrane ${ }^{6}$. In plants, there are two distinct pathways for galactolipid biosynthesis in the plastid and the ER, which are generally known as the "prokaryotic pathway" and

\section{(c) The Author(s) 2020}

(c) (i) Open Access This article is licensed under a Creative Commons Attribution 4.0 International License, which permits use, sharing, adaptation, distribution and reproduction in any medium or format, as long as you give appropriate credit to the original author(s) and the source, provide a link to the Creative Commons license, and indicate if changes were made. The images or other third party material in this article are included in the article's Creative Commons license, unless indicated otherwise in a credit line to the material. If material is not included in the article's Creative Commons license and your intended use is not permitted by statutory regulation or exceeds the permitted use, you will need to obtain permission directly from the copyright holder. To view a copy of this license, visit http://creativecommons.org/licenses/by/4.0/. 
"eukaryotic pathway", respectively. Owing to the substrate preference of lysophosphatidic acyltransferase, galactolipids generated from the "prokaryotic pathway" tend to carry C16 fatty acids at the sn-2 position, while the "eukaryotic pathway" generates galactolipids with $\mathrm{C} 18$ fatty acids at the sn-2 position. Therefore, plants are sometimes classified accordingly as "16:3 plants" or "18:3 plants".

In plants, all lipid compounds share acyl-CoA pools in the cytosol ${ }^{7}$. However, the carbon distribution between different lipid classes could be rigorous or flexible ${ }^{8}$. Among most of the known cer (eceriferum) mutants with varying degrees of reduction in wax levels, there seems to be an increase in the flux of precursors into an alternative branch pathway ${ }^{9}$, and fatb mutants, with low levels of wax, showed dramatic changes in the formation rate of both prokaryotic and eukaryotic lipids ${ }^{10}$. Thus, knowledge of the variations in lipid contents will provide deep insight into the defense strategy that plants adopt in terms of lipid metabolic pathways. However, few studies have focused on how lipids are altered in cuticle mutants of the model plant Arabidopsis, and in crop species, such as citrus, where the cuticle may be highly correlated with industrial value, the number of studies is even smaller.

The promotion of membrane lipid levels may be an effective route to enhance plant performance. Furthermore, an increase in plastid lipids has some unique advantages. First, increased levels of plastid lipids could contribute to highly curved membrane regions for the photosystems, thereby positively affecting the capture of light energy and its conversion ${ }^{11,12}$. Second, previous reports have demonstrated that enhanced tolerance to cold, salt and drought stress corresponds to a higher proportion of highly unsaturated fatty acyls ${ }^{13,14}$, which is one characteristic of plastid lipids.

Modifications of plastid lipids have been well documented $^{15}$. The phospholipase A1 (PLA1) gene family, which functions in the hydrolyzation of the sn-1 position of glycerol backbones, is known to play critical roles in membrane lipid maintenance and synthesis of signaling mediators, such as jasmonic acid (JA), using hydrolyzed products $^{16,17}$. To date, members from two PLA1 gene families have been reported to be involved in JA biosynthesis, namely, the DEFECTIVE ANTHER DEHISCENCE 1-like (DAD1-Like) gene family, including DAD1 and DONGLE (DGL), and the PLASTID LIPASE (PLIP) gene family, including PLIP2 and PLIP3 ${ }^{18-20}$. However, controversy regarding their roles still exists: DGL localizes to lipid droplets rather than to chloroplasts, and DAD1 only shows high expression in the late wound response ${ }^{18}$. Although the lipase-catalyzed reaction is regarded as the rate-limiting step in JA synthesis ${ }^{21}$, little is known about which lipase genes are involved in the formation of endogenous JA from plastid lipids in citrus.
To date, most molecular studies related to lipids in citrus have only focused on the cuticle ${ }^{3,4,22}$, without highlighting the importance of membrane lipids. Based on the previously reported glossy mutant of the "Newhall" navel orange ${ }^{4}$, we performed lipidomic and transcriptomic analysis on both immature and mature stages of this glossy mutant (MT) and the wild-type "Newhall" navel orange (WT) to ascertain the carbon exchanges between wax and plastid lipids. We also attempted to elucidate the possible reasons underlying the enhanced JA levels in MT by the functional characterization of three upregulated lipase genes. This study aimed to improve the understanding of quality maintenance in citrus fruits and provide new insights into the molecular mechanisms underlying the naturally occurring variations of lipid pools in citrus.

\section{Results}

\section{Wax component contents decreased in MT throughout fruit development}

A glossy phenotype became apparent during the fruit ripening process of MT (Fig. 1a). To further verify how wax contents were altered in developmental stages, we selected three stages: 90 days after anthesis (DAA), 150 DAA and 210 DAA, when fruits started to expand, began to ripen and became fully ripe, respectively. Among the wax components of the "Newhall" navel orange, aldehydes accounted for the largest proportion, followed by that of alkanes, FA, and alcohols ${ }^{23}$. Most aliphatic compounds showed a decreasing, and then sharply increasing, trend, but a continuous accumulation of aldehyde components was observed in both WT and MT (Fig. 1c). All aliphatic compounds in the wax were present in lower concentrations in MT than in WT across the entire developmental process, with slight differences at $90 \mathrm{DAA}$ and increasing differences with increasing fruit maturation. In the case of aldehydes, we first observed a 21-fold change (FC) decrease in MT $\left(0.05 \mu \mathrm{g} / \mathrm{cm}^{2}\right)$ compared with that in WT $\left(1.03 \mu \mathrm{g} / \mathrm{cm}^{2}\right)$ at $90 \mathrm{DAA}$. At $150 \mathrm{DAA}$, the FC was 43 $\left(0.04 \mu \mathrm{g} / \mathrm{cm}^{2}\right.$ vs $\left.1.55 \mu \mathrm{g} / \mathrm{cm}^{2}\right)$, and at $210 \mathrm{DAA}$, the FC peaked at $88\left(0.082 \mu \mathrm{g} / \mathrm{cm}^{2}\right.$ vs $\left.7.78 \mu \mathrm{g} / \mathrm{cm}^{2}\right)$. Moreover, the proportion of alkanes in MT was reduced by $70 \%$ compared with than in WT, and that of alcohols was reduced by $80 \%$ at 210 DAA.

\section{Glycerol lipids increased in MT}

Wax biosynthesis is one direction of carbon flux from acyl-CoA pools. Thus, the blocking of carbon flux toward wax biosynthesis may have certain feedback effects on the acyl-CoA pools and may subsequently alter membrane lipid metabolism in MT. To verify this hypothesis, we carried out lipidomic profiling on flavedo tissues peeled from fruits at 150 DAA and 210 DAA, when relatively large differences in wax contents were observed (Fig. 1). A total of 13 classes of 


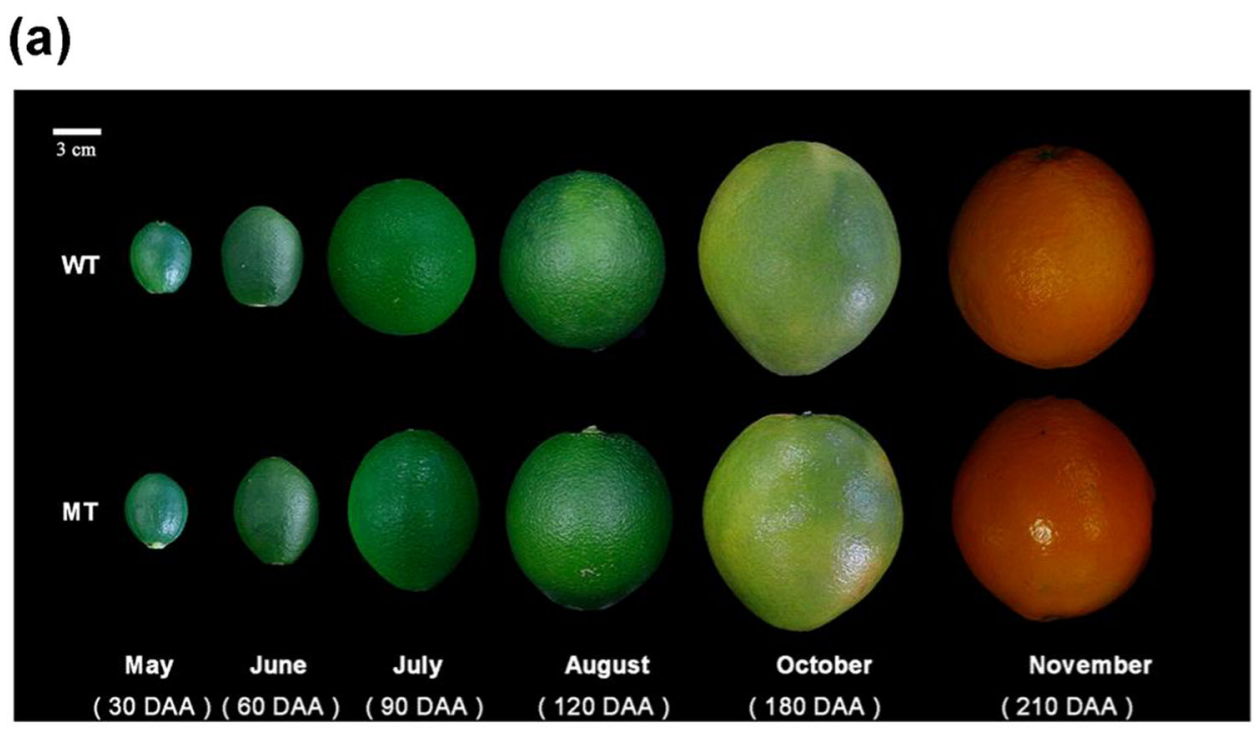

(b)

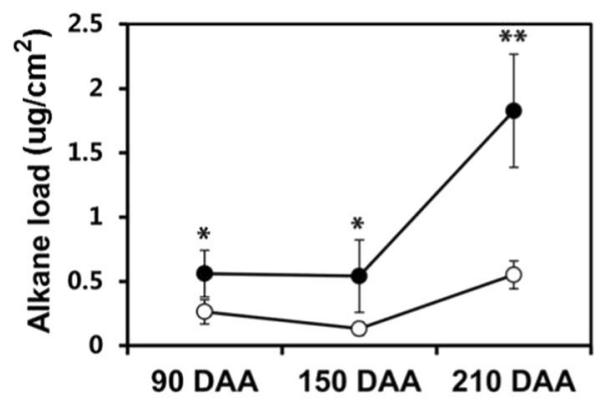

(d)

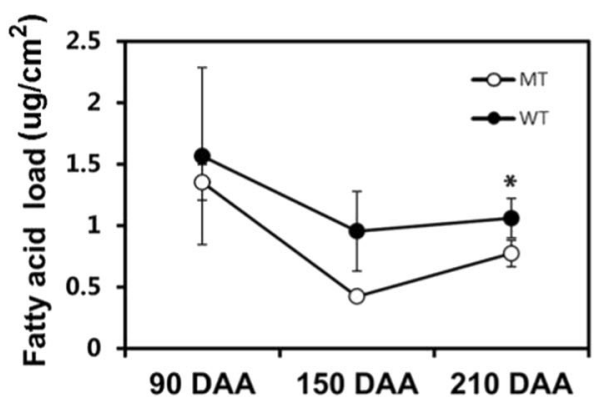

(c)

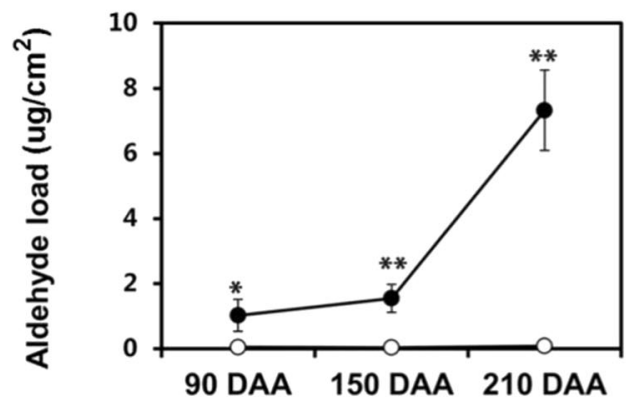

(e)

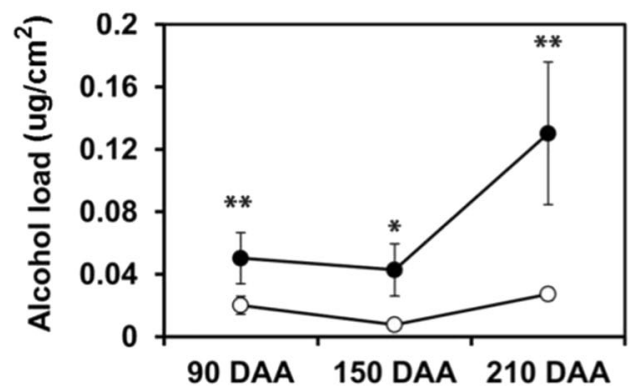

Fig. 1 Glossy phenotype and wax synthesis with increasing maturation in MT and WT. a Gradual, obvious glossy phenotype of MT compared with that of WT with increasing fruit maturation. DAA days after anthesis, MT mutant type, WT wild type. b-e Levels of main wax classes in flavedos of WT and MT in the three stages analyzed. $\mathbf{b}$ Alkanes, $\mathbf{c}$ aldehydes, $\mathbf{d}$ cuticular fatty acids, and e primary alcohols. The level of each wax class was the sum of all subclasses. Values are the average of three biological replicates \pm standard deviation $(\mathrm{SD})(n=3)$. An asterisk $\left(^{*}\right)$ indicates a $t$-test $p$ value $\leq$ 0.05 and double asterisks ${ }^{* *}$ indicate a $t$-test $p$ value $\leq 0.01$. More detailed information on the molecular species is available in Supplementary Fig. S1

glycerolipids containing 219 kinds of species were identified and quantified, including 46 TAGs (triacylglycerols), 14 DAGs (diacylglycerols), 20 PCs, 24 PEs, 13 PGs, 14 PIs (phosphatidylinositols), 26 PSs (phosphatidylserines), 12 PAs (phosphatidic acid), 17 MGDGs, 17 DGDGs, 6 LysoPCs (lysophosphatidylcholines), 5 LysoPEs (lysophosphatidylethanolamines), and 5 LysoPGs (lysophosphatidylglycerols) (Supplementary Table S1). As indicated by hierarchical clustering analysis (Fig. 2a), the metabolism of phospholipids in MT showed a distinct pattern from that in WT. Higher lipid accumulations in MT than in WT were observed at both stages according to the relative contents of 
(a)

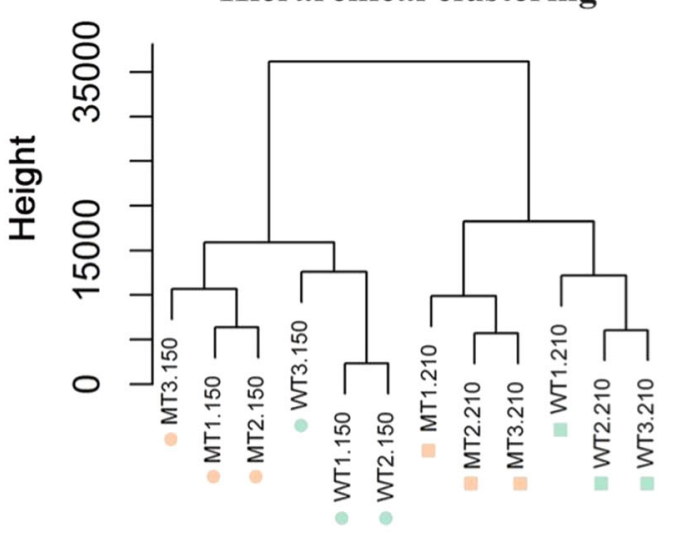

(b) Total phosphalipid

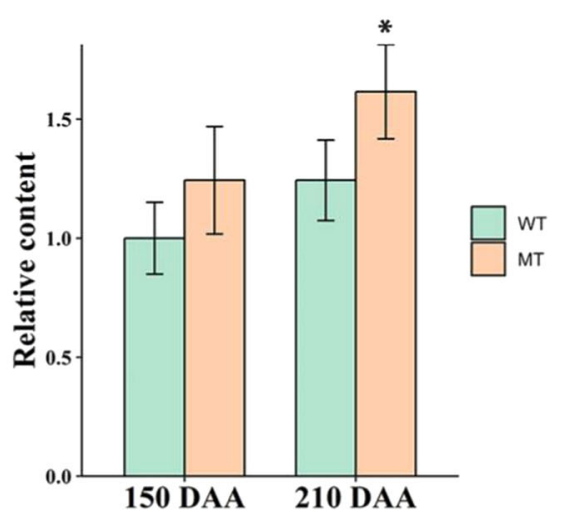

(c)

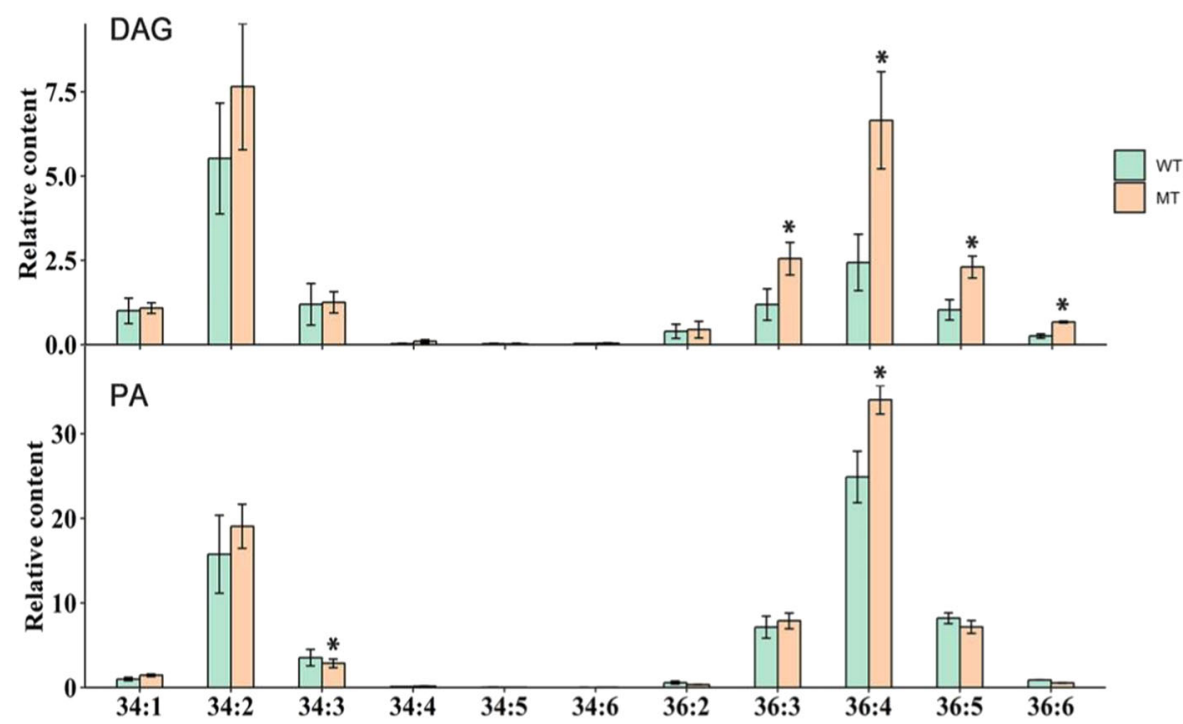

Fig. 2 Contents of glycerophospholipids in MT and WT at 150 DAA and 210 DAA. a Cluster analysis of all samples for lipid analysis in WT and MT based on average hierarchical clustering. Numbers represent the days after anthesis; WT wild type, MT mutant type. b Relative content of total phospholipids observed at 150 and 210 DAA. Corrected areas of all phospholipid classes in each replicate were summed and normalized by the average value number WT at 150 DAA. Bars are the average of three normalized biological replicates \pm standard deviation (SD, $n=3)$. c Relative contents of two main lipid precursors, PAs and DAGs, at 210 DAA. The corrected area of each subclass was summed and normalized by the average number of 34:1 carbon molecules in DAGs and PAs in WT. Bars are the average of three normalized biological replicates at 210 DAA \pm standard deviation (SD, $n=3$ ). An asterisk $\left(^{*}\right)$ indicates a t-test $p$ value $\leq 0.05$. DAGs diacylglycerols, PAs phosphatidic acids

all lipid classes (Fig. 2b), particularly at 210 DAA. The higher lipid accumulation in MT at 210 DAA was further validated with samples harvested in another year (2017) (Supplementary Fig. S2b and Supplementary Table S2).

Phosphatidic acids are essential intermediates in the synthesis of diverse glycerolipids. Here, we found a similar trend in the variations of levels and carbon species between PAs and DAGs. For example, 36:4 and 34:2 carbon molecules were coincidently dominant in both lipid classes and were simultaneously enhanced in MT (Fig. 2c), and 36:4 PA showed a strong correlation (Pearson correlation coefficient $(\mathrm{PCC}) \geq 0.7, p \leq 0.05$ ) with 36:3,4,5-carbon DAGs (Fig. 3e and Supplementary Table S3). Similar results were obtained in 2017 in which 34:2, 36:3, and 36:4 carbon molecules were shown to account for a larger proportion of both PAs and DAGs, and all of them showed high accumulations in MT (Supplementary Fig. S2c). These results suggested a tight metabolic connection between PAs and DAGs in the flavedo of "Newhall" navel oranges.

Further analysis of DAGs showed that the accumulations of 36 highly unsaturated carbon molecules, including 36:3, 36:4, 36:5, and 36:6, were all significantly ( $t$-test $p$ value $\leq 0.05)$ elevated in MT relative to those in WT at 
(a)

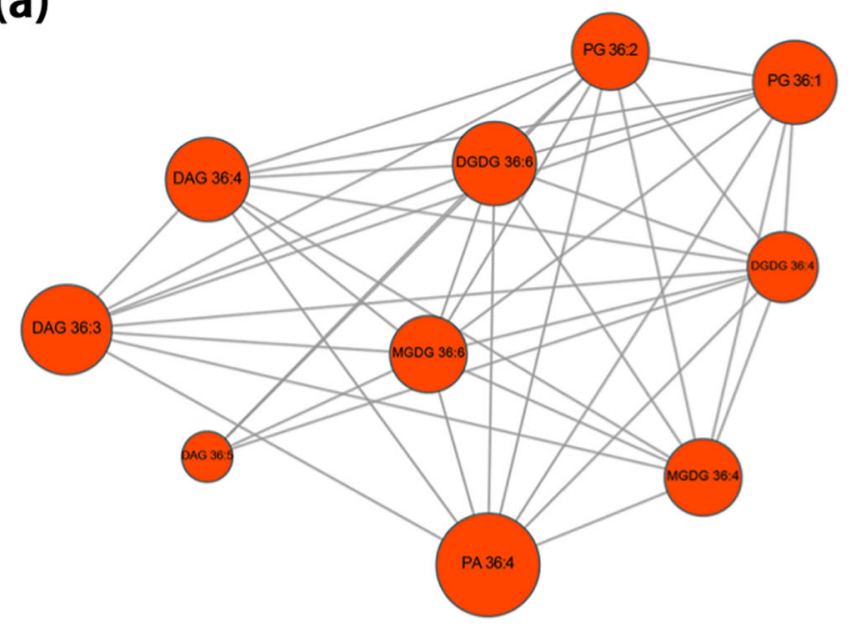

(b)

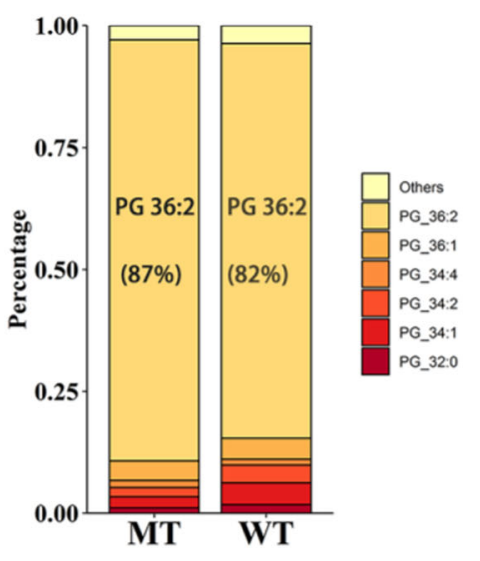

\section{(c) MGDG}
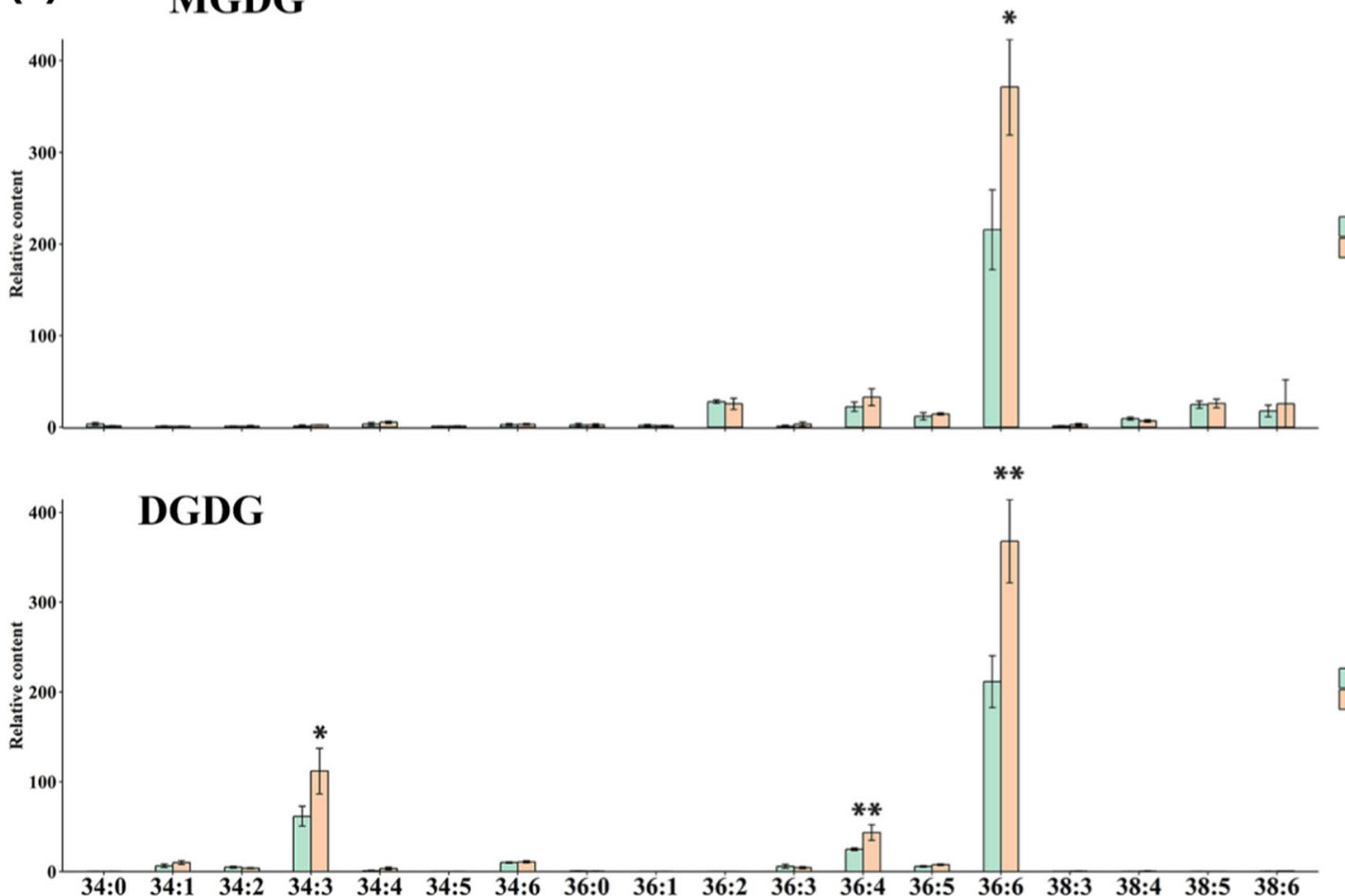

Fig. 3 Variations in the main subclasses of plastid lipids at 210 DAA. a Network illustrating the highly correlated 36-carbon lipid compounds. Lipid pairs with PCC (Pearson correlation coefficient) values over 0.70 and $p$ values $<0.05$ are shown. The bubble size indicates the number of correlated metabolites. $\mathbf{b}$ Proportions of subclasses in the PG class. Subclasses accounting for more than 1\% in each class at 210 DAA are displayed in the diagram. Other lipids are classified into "Others," and those with higher proportions are marked on the diagram. c Relative contents of two main chloroplast lipids, MGDGs and DGDGs, at 210 DAA. The corrected area of each subclass was summed and normalized by the average number of 34:2 and 34:4 carbon molecules in MGDGs and DGDGs in WT, respectively. Bars are the average of three normalized biological replicates at 210 DAA \pm standard deviation (SD, $n=3)$. An asterisk $\left(^{*}\right)$ indicates a $t$-test $p$ value $\leq 0.05$ and double asterisks $\left(^{* *}\right)$ mean $t$-test $p$ value $\leq 0.01$. MGDGs

monogalactosyldiacylglycerols, DGDGs digalactosyldiacylglycerols, PG phosphatidylglycerol

150 DAA and 210 DAA (Fig. 2c). Given that 36-carbon DAG could provide skeletons for the biosynthesis of 36carbon chloroplast galactolipids, it was interesting to observe that 36:6-carbon MGDG and 36:6-carbon DGDG were significantly increased ( $t$-test $p$ value $\leq 0.05$ ) in MT, and 36:3,4,5-carbon DAGs showed a strong correlation with 36-carbon plastid lipids, such as 36:6 MGDG, 36:6 DGDG, and 36:2 PG (Fig. 3a and Supplementary Table 
S3). In addition, the dominant proportion of 36:6 molecules in MGDGs and DGDGs (over 60\%) in WT and MT (Fig. 3c) demonstrated that the "Newhall" orange mainly depends on the eukaryotic pathway for galactolipid synthesis, similar to most reported seed plants, indicating that "Newhall" orange is a typical "18:3 plant". DAGs could also provide skeletons for the biosynthesis of TAGs. However, less obvious changes in the levels of TAG subclasses were observed between WT and MT at both 150 DAA and 210 DAA (Supplementary Fig. S3). Interestingly, 36:2 carbon species were dominant in PGs (Fig. $3 \mathrm{~b})$ and were significantly upregulated in MT, also suggesting that the ER is the main source of carbon for PG biosynthesis. Similar results were observed in samples harvested in 2017 (Supplementary Fig. S4). Overall, our data showed that plastid lipids, together with the phospholipid precursors PAs and DAGs, were increased in MT compared with those in WT.

The increase in the three most abundant subclasses of lyso-PGs, the possible hydrolyzation products of glycerophospholipids (Fig. $4 \mathrm{a}-\mathrm{c}$ ) at the mature stage, suggested a possible induction of the phospholipid hydrolyzation pathway in MT. Given that the biosynthesis of JA originates from the degradation of chloroplast lipids, we then tested the JA level at 210 DAA. JA was significantly accumulated ( $t$-test $p$ value $\leq 0.05)$ in the flavedo and juice sacs of MT (Supplementary Fig. S5), demonstrating that JA biosynthesis was strongly affected by the altered lipid metabolism in MT in 2014.

\section{Transcriptomic differences in the flavedo between MT and WT during developmental stages}

To further investigate the differences in molecular biological regulation between MT and WT, a transcriptomic analysis was performed on the fruit flavedo harvested at 150 DAA and 210 DAA. Principal component analysis indicated that the differences between the two stages were larger than those between WT and MT (Supplementary Fig. S6a). Thus, we mainly focused on the differentially expressed genes (DEGs) between WT and MT at the same stage. Similar to the gradually larger differences in the lipidomic data, the transcriptomic data revealed that the proportion of DEGs increased with increasing fruit maturation. The number of upregulated genes was smaller at the immature stage and then became similar to that of downregulated genes at the mature stage (Fig. 5a). Similar results were observed in the transcriptomic data at 210 DAA from fruit grown in 2017 (Supplementary Fig. S6b, c). These DEGs were significantly enriched in 24 KEGG pathways. Metabolic pathways such as "photosynthesis", "photosynthesisantenna proteins", and "carbon fixation in photosynthetic organisms", were closely associated with photosynthesis, while " $\alpha$-linoleic acid metabolism" and "fatty acid metabolism" were closely related to lipid metabolism (Fig. 5b). To identify pathways involved in plastid lipid metabolism in "Newhall" navel oranges, we generated correlation networks based on lipidomic and transcriptomic data (Supplementary Table S4). Given the dominant proportion and high correlation of 36:6 MGDG and DGDG, and 36:2 PG (Fig. 3), we used the three lipid species to represent the whole plastid lipid profile and focused on overlapping genes that positively correlated with them. A total of 744 genes showed a strong positive correlation with plastid lipids. Among the significantly enriched GO terms of the 744 genes, we found the "JA metabolic process" and "pyruvate metabolism" (Fig. 5c).

To confirm the lipid-related changes at the transcriptional level, we focused on the variations of a compiled list of 93 lipid-related genes among all the up- or downregulated genes (Supplementary Table S5), which were screened mainly based on a homologous BLAST search against previously released genes in the Arabidopsis lipid gene database (http://aralip.plantbiology.msu.edu/ pathways/pathways) reported by Li-Beisson et al. ${ }^{24}$. Table 1 presents the lipid genes and some representative transcriptional factors whose expression levels correspond well with the changes in metabolites. Among them, genes that showed a high correlation with MGDG 36:6 were also indicated.

\section{Variations in lipid-related genes across developmental stages}

Based on the results of the lipidomic and transcriptomic analysis, a schematic diagram was generated to reveal the most significantly altered lipid pathways in MT compared with those in WT (Fig. 6a). Consistent with the gradual decrease in the content of aliphatic wax compounds in $\mathrm{MT}$, the expression levels of gene families involved in wax biosynthesis pathways also displayed a constant decreasing trend in MT (Fig. 6b). However, a number of them showed a high correlation with MGDG 36:6. Fatty acylACP thioesterase B (FATB), LACS1 and LACS2 (longchain acyl-CoA synthetase), which are responsible for providing precursors for wax synthesis, exhibited downregulated expression in MT, and all three genes showed a negative correlation with 36:6 MGDG and 36:6 DGDG (Table 1 and Supplementary Table S4). The expression levels of three of the five KCSs ( $\beta$-ketone CoA synthases) were suppressed in MT in both the 2014 or 2017 data (Supplemental Table S5); however, the remaining two KCSs, KCS4 and KCS2b, showed upregulated profiles and were positively correlated with all three plastid lipids. All six fatty acid reductases (FARs) were significantly downregulated in MT, and CsFAR2, together with CER3, was also downregulated in the 2017 transcriptome data and in another glossy mutant of the "Newhall" orange named 

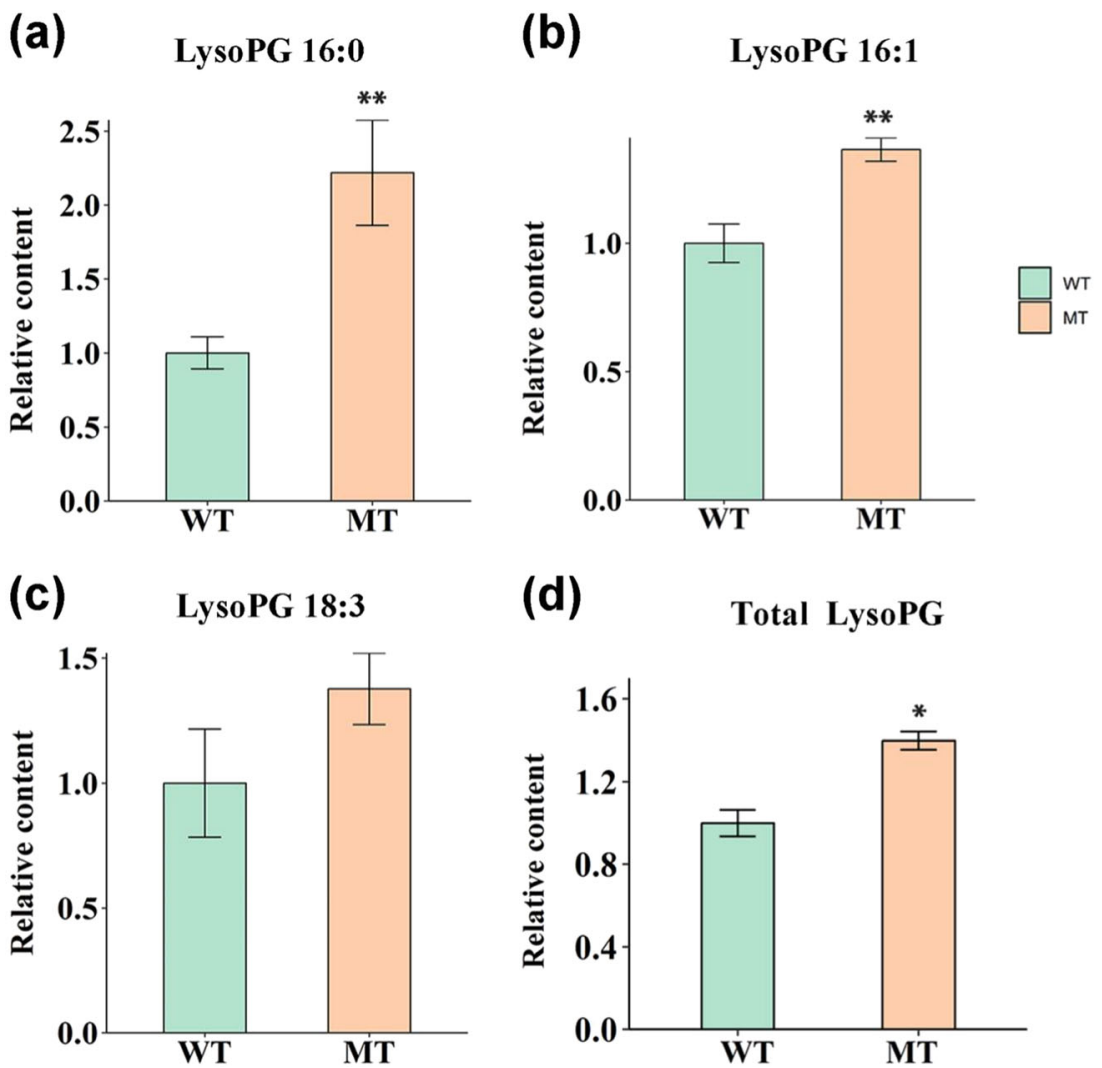

(d)

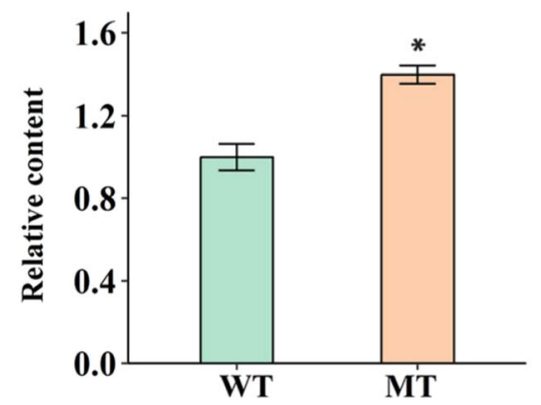

Fig. 4 Contents of plastid lipid degradation products, lysoPGs at 210 DAA. a-c Relative content of three main lysoPG subclasses in MT compared with that in WT at 210 DAA. a LysoPG 16:0; b LysoPG 16:1; c LysoPG 18:3. d Relative content of total lyso PGs in MT compared with WT on 210 DAA. Corrected areas of each lysoPG subclass were summed and normalized by the average number in WT. Bars are the average of three normalized biological replicates \pm standard deviation $(S D, n=3)$. An asterisk $\left(^{*}\right)$ indicates a $t$-test $p$ value $\leq 0.05$ and double asterisks $\left({ }^{* *}\right)$ indicate a $t$-test $p$ value $\leq 0.01$

"Ganqi 3" (Table 1), which was consistent with the reduced alcohol and aldehyde levels ${ }^{25}$.

Fatty acid desaturase 2 (FAD2) initiates the formation of ER-derived plastid galactolipid biosynthesis ${ }^{26}$. We found that CsFAD2 showed a more obvious upregulation at 210 DAA, and the gene showed a positive correlation with MGDG and DGDG 36:6. It is worth noting that upregulation of the marker gene FAD2 was further confirmed by transcriptome data from 2017. Phospholipase D (PLD) and phospholipase $C$ (PLC) gene families are involved in the conversion of desaturated PCs into PAs and DAGs. Two PLDs, including PLD $\alpha 1$ and PLD 1 , were upregulated in MT at the mature stage, and CsPLD 1 (Cs1g12600) exhibited much higher expression and thus may play a more important role than PLD 11 (Supplementary Table S5). Among all three NPCs identified, CsPLC3 showed a 1.1- and 0.62-FC increase at 210 DAA in the 2014 and 2017 data, respectively, possibly contributing to an increase in DAGs hydrolyzed from PCs. Furthermore, enhanced plastid galactolipid biosynthesis in MT was also supported by the upregulation of FAD5 and FAD7 in the 2-year transcriptome data, both of which are regarded as key genes influencing the formation of MGDGs, and FAD5 showed a highly positive correlation with plastid lipids.

Consistent with the increased JA level in MT, genes involved in lipoxygenation pathways were upregulated. Three of the four DAD1 orthologs (CsDALL2, CsDALL4, and CsDAD1), four lipoxygenases, two allene oxide synthases (AOSs), four oxo-phytodienoic acid reductases, and two JA carboxyl methyltransferases (JCMTs) were upregulated at the mature stage. Notably, the upregulation of most of the abovementioned genes (12 out of 18) was further confirmed by the transcriptome data from 2017. Interestingly, we found that CsDAD1, CsDALL2, CsAOS (Cs3g24230), and JCMTa (Cs3g25140) were strongly correlated with plastid lipids. In addition, as a signaling hormone, JA at relatively high levels could induce the JA signaling pathways, and this induction could be signified by the degradation of JAZ proteins (JAZMONATE ZIM DOMAIN proteins) ${ }^{19}$. Indeed, we identified that three JAZ homologs, CsJAZ1(a) (Cs1g17210), CsJAZ1(b) (Cs1g17220), and CsJAZ8 (Cs2g03240), were 

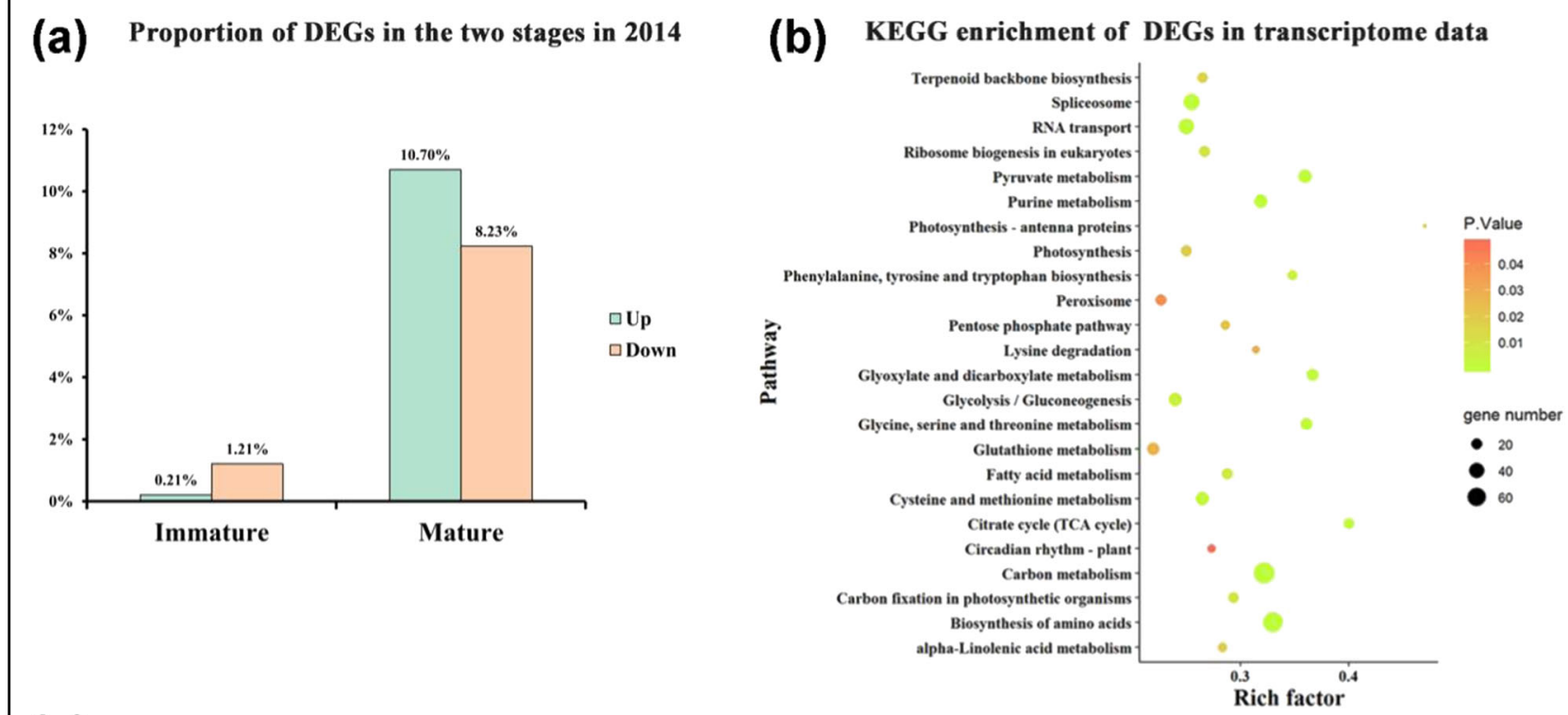

(C) GO enrichment of gene sets positively correlated to plastid lipids

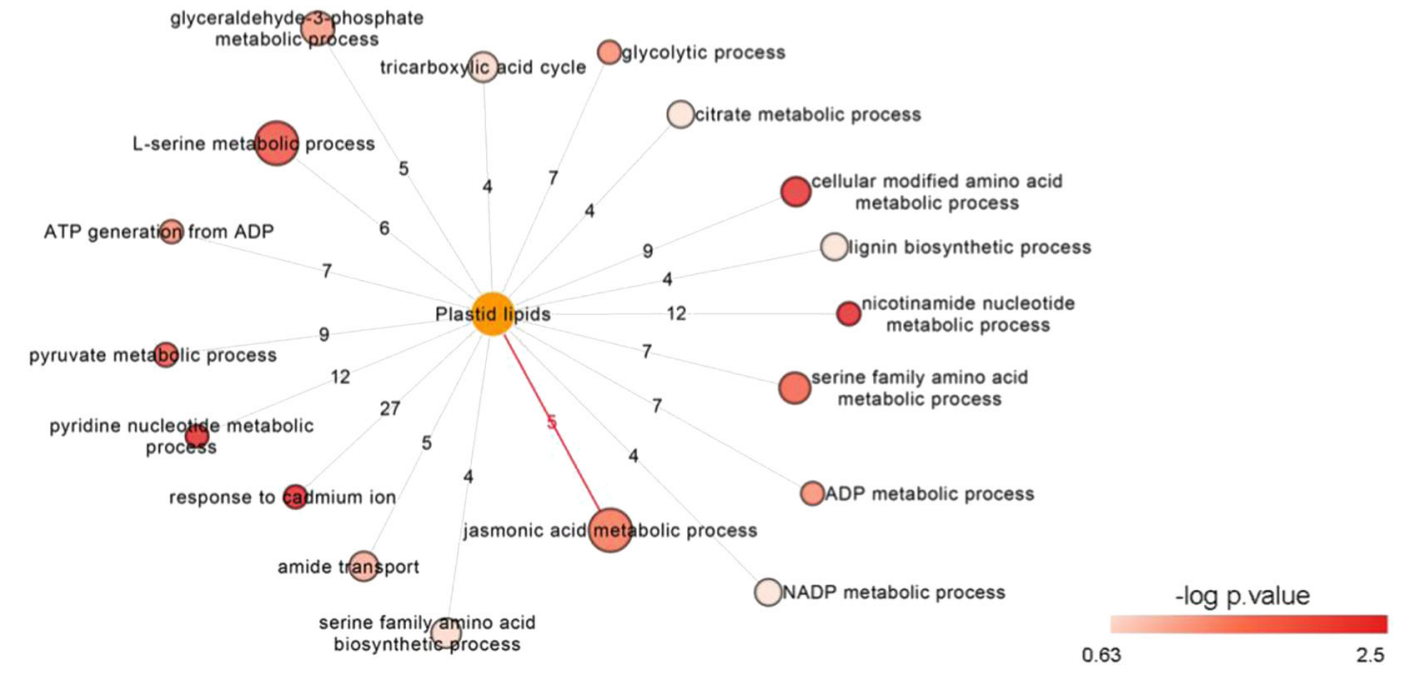

Fig. 5 Transcriptomic analysis of WT and MT. a Proportions of DEGs in the two developmental stages. The proportions were calculated as the number of DEGs/number of expressed genes in the respective month. $\mathbf{b}$ The first 20 enriched pathways according to KEGG analysis of the total DEGs characterized in both immature and mature stages. The size of the bubble indicates the input gene number in the pathway, and the color indicates the $p$ value. c Significantly enriched gene ontology $(\mathrm{GO})$ terms (metabolic process) of genes that positively correlated $(P C C \geq 0.7, p \leq 0.05)$ with the three main plastid lipids, MGDG 36:6, DGDG 36:6, and PG 36:2. Genes showing a high positive correlation with each of the three plastid lipids were selected, intersected and used for GO enrichment analysis. The color of the bubble indicates enrichment factor, and the color scale indicates the $p$ value. The numbers of input genes are indicated on the lines

significantly downregulated in MT (Supplementary Table S5), and interestingly, CsJAZ1(a) showed a strong negative correlation with plastid lipids.

\section{Expression patterns of lipid-related transporters and transcriptional factors}

The ATP-binding cassette transporter G family (ABCG) contributes to the synthesis of wax, and three out of the seven ABCGs were consistently downregulated in MT in 2 analysis years. Among them, ABCG 32 is required for the biosynthesis and accumulation of cuticle lipids in both maize and Arabidopsis ${ }^{27,28}$, and ABCG15 is the closest homolog of CER $5^{29}$, an indispensable gene for wax formation in Arabidopsis. Interestingly, both of the genes showed a highly negative correlation with plastid genes. Phospholipid exchange between different organelles also requires lipid transporters, and the protein TRIGALACTOSYLDIACY-L GLYCEROL 1 (TGD1) transfers PA from the ER to the chloroplast ${ }^{30}$. Two homologs of TGD1 were characterized, with CsTGD1 (a) 
Table 1 Summary of key genes involved in the alteration of lipid metabolism pathways

\begin{tabular}{|c|c|c|c|c|c|}
\hline \multirow[t]{2}{*}{ Gene ID } & \multicolumn{3}{|c|}{$\log 2$ ratio (MT/WT) } & \multirow[t]{2}{*}{ PCC (MGDG) } & \multirow[t]{2}{*}{ Annotation } \\
\hline & 150DAA (2014) & 210DAA (2014) & 210DAA (2017) & & \\
\hline \multicolumn{6}{|l|}{ Wax formation } \\
\hline $\operatorname{Cs} 2 g 13450^{a}$ & & -1.98 & & $-0.71^{*}$ & Fatty acyl-ACP thioesterases B \\
\hline Cs5g28330 & & -1.36 & -1.71 & $-0.65^{*}$ & Long-chain acyl-CoA synthetase 2 \\
\hline orange1.1t01720 & & -0.97 & -1.28 & $-0.86^{* *}$ & Long-chain Acyl-CoA synthetase 1 \\
\hline Cs9g07970 & & -1.32 & -1.26 & - & Ketoacyl-CoA synthase 2(a) \\
\hline Cs6g02360 & & 1.02 & & $0.88^{* *}$ & Ketoacyl-CoA synthase 2(b) \\
\hline orange $1.1 \mathrm{t} 02246^{\mathrm{a}}$ & & -0.98 & -0.78 & - & Fatty acyl-CoA reductase/FAR2 \\
\hline Cs5g15350 & & 0.00 & -2.67 & $-0.77^{* *}$ & Fatty acyl-CoA reductase 3 \\
\hline Cs4g02580 & -2.51 & -1.98 & -2.70 & - & CER3 protein \\
\hline Cs3g10550 & & 0.83 & & $0.88^{* *}$ & CER2 protein \\
\hline orange $1.1 \mathrm{t} 02321^{\mathrm{a}}$ & & -1.42 & -2.29 & $-0.85^{* *}$ & ABCG 32 \\
\hline Cs4g17100 & & -0.95 & -0.73 & $-0.78^{* *}$ & ABCG 16 \\
\hline \multicolumn{6}{|c|}{ Eukaryotic PC turnover } \\
\hline orange1.1t02241 & & 2.12 & 0.96 & $0.71^{*}$ & Oleate desaturase 2/FAD2 \\
\hline Cs1g12430 & & 1.27 & 1.94 & $0.78^{* *}$ & Acyl-CoA desaturase-like/FAD5 \\
\hline Cs6g08600 & & 0.79 & 0.64 & - & Linoleate desaturase/FAD7 \\
\hline Cs7g17090 & & 2.43 & & $0.89^{* *}$ & Glycerol-3-phosphate Acyltransferase 1 \\
\hline Cs5g17310 & & & -0.53 & $-0.67^{*}$ & Lysophospholipid acyltransferase \\
\hline Cs4g 10970 & & & 1.00 & -0.69 & 1-acylglycerol-3-phosphate acyltransferase 1 \\
\hline Cs1g12600 & & 1.49 & & - & PL Dalpha 1 \\
\hline Cs7g15610 & & 1.10 & 0.62 & $-0.67^{*}$ & Phospholipase C (Nonspecific) C3 \\
\hline $\operatorname{Cs} 8 g 20240^{a}$ & & -1.50 & -1.77 & $-0.76^{*}$ & Phosphoinositide-specific phospholipase C4 \\
\hline \multicolumn{6}{|l|}{ JA biosynthesis } \\
\hline Cs2g30480 & & 2.60 & 2.03 & $0.80^{* *}$ & Acylhydrolase (DAD1-like) \\
\hline Cs1g03190 & & 2.06 & & $0.80^{* *}$ & Acylhydrolase (DAD1-like) \\
\hline Cs5g08030 & & -0.95 & & $-0.70^{*}$ & Acylhydrolase (DAD1-like) \\
\hline orange1.1t04376 & & 1.77 & & - & LOX2C \\
\hline Cs2g21000 & & 2.43 & 0.71 & - & HPL1 \\
\hline Cs5g17900 & & 1.23 & 1.13 & - & OPR \\
\hline Cs5g17920 & -1.08 & 1.52 & 0.61 & - & OPR \\
\hline Cs3g24230 & & 1.22 & 0.59 & $0.85^{* *}$ & Allene oxide synthase \\
\hline Cs3g25140 & & 3.91 & 4.80 & $0.84^{* *}$ & Jasmonic acid carboxyl methyltransferase \\
\hline \multicolumn{6}{|l|}{ Others } \\
\hline Cs1g13620 & & 1.91 & 0.56 & $0.91^{* *}$ & ABCG 40 \\
\hline Cs7g28090 & & 1.29 & -0.52 & $0.87^{* *}$ & ABCG 6 \\
\hline Cs8g15960 & & -0.78 & -1.17 & $-0.67^{*}$ & ABCG 3 \\
\hline Cs5g23300 & & 0.90 & -0.54 & $0.91^{* *}$ & Trigalactosyl diacylglycerol 1(a) \\
\hline Cs6g09960 & & 3.75 & 3.46 & $0.82^{* *}$ & Lipid transfer protein type 1 \\
\hline
\end{tabular}


Table 1 continued

\begin{tabular}{|c|c|c|c|c|c|}
\hline \multirow[t]{2}{*}{ Gene ID } & \multicolumn{3}{|c|}{$\log 2$ ratio (MT/WT) } & \multirow[t]{2}{*}{ PCC (MGDG) } & \multirow[t]{2}{*}{ Annotation } \\
\hline & 150DAA (2014) & 210DAA (2014) & 210DAA (2017) & & \\
\hline Cs7g06120 & -1.19 & -2.12 & & - & SHN ESE3 \\
\hline Cs6g21530 & & -1.85 & -1.30 & - & Myb domain protein 16 \\
\hline Cs5g30520 & & 1.96 & 0.98 & $0.91^{* *}$ & Pyruvate Kinase \\
\hline Cs1g17210 & & -1.31 & & $-0.81^{* *}$ & JASMONATE-ZIM-DOMAIN PROTEIN 1 \\
\hline
\end{tabular}

PCC Pearson correlation coefficient. ${ }^{*} p$ value $\leq 0.05,{ }^{* *} p$ value $\leq 0.01$

a Genes also found in the other two datasets of "Newhall" navel oranges.

being upregulated at the mature stage and showing high correlation with plastid lipids.

The precise regulation of lipid biosynthesis occurs at least partly at the transcriptional level. Wax-related TFs, such as MYB16 and ESE3, a homolog of the SHN family, were also significantly downregulated in MT. In addition to the known TFs in lipid-related pathways, a full list of transcriptional factors with significant differential expression and high correlation with plastid lipids is provided in Supplementary Table S6. Among these differentially expressed TFs, NAC072, which has relatively high expression and is involved in the COI1-JAZ-MYC2 JA signaling pathway in Arabidopsis ${ }^{31}$, was upregulated and showed a high correlation with plastid lipids.

\section{Subcellular location and transient overexpression of the three PLA1 homologs $N$. benthamiana}

JA biosynthesis is closely related to plastid lipids. To determine the key lipases involved in plastid lipid degradation and JA formation, we performed a protein sequence BLAST search against phospholipase A family genes reported in Arabidopsis ${ }^{16,20,32}$. A total of 37 putative lipases were identified (Supplementary Table S7). Phylogenetic analysis of these genes revealed that three PLA1 genes were grouped within the DAD1-like gene family (Fig. 7a and Supplementary Fig. S7a). Among them, the two genes CsDAD1 and CsDALL2 showed a positive correlation with plastid lipids (Table 1). We further investigated the subcellular location of the three significantly upregulated DAD1-like genes and observed that all of them were localized in the chloroplast (Fig. 7b), which is consistent with their putative functions in liberating plastid FAs from chloroplasts. To experimentally verify the function of these genes as chloroplast lipases, we performed transient overexpression of the three lipases in Nicotiana benthamiana, which has been proven to be an efficient method for the functional characterization of lipid-related genes ${ }^{33,34}$. Relative increases over 30 -fold were observed in CsDAD1, CsDALL2, and CsDALL4 overexpression lines $(\mathrm{OE})$ after infiltration (Supplementary Fig. S8). Among all the chloroplast lipid classes (proportion over $0.5 \%$ ) in CsDALL4 OE lines, only the content of DGDG 36:2 decreased. However, in the CsDAD1 OE lines, a number of 34-carbon chloroplast lipids were significantly ( $t$-test $p$ value $\leq 0.05, n=3$ ) hydrolyzed, especially 34:2 PG, accounting for approximately 33\% of all PGs and 34:6 MGDG, accounting for $\sim 27 \%$ of all MGDGs, which were significantly decreased by $33.84 \%$ and $31.26 \%$, respectively (Fig. 7c, d and Supplementary Table S8). In the plastid lipids characterized in CsDALL2 OE lines, we observed a significant decrease in the levels of both 36- and 34-carbon lipids. For example, two 34-carbon PGs, 34:1, 34:2 (accounting for $2.6 \%$ of all PGs) and 36:4.

MGDG (accounting for $\sim 2.0 \%$ of all MGDGs) were significantly decreased by $47.4 \%, 26.9 \%$ and $17.9 \%$, respectively (Fig. 7e, f). To verify whether the hydrolyzed FAs contributed to JA formation, we tested the levels of JA in the three overexpression lines. The level of JA in EV lines was similar to that previously reported in tobacco ${ }^{35}$, and the CsDAll4 OE lines had almost equal amounts of JA ( 96.6 vs $81.4 \mathrm{ng} / \mathrm{g}$ in CsDALL4 OE and EV, respectively), indicating that the gene exerts little influence on JA biosynthesis. However, we observed 19- and 8-fold increases in JA levels in the CsDAD1 and CsDALL2 overexpression lines, respectively, suggesting that the two genes have a large influence on JA formation (Fig. 7g).

\section{Discussion}

Reallocation of carbon flux from cuticular wax into plastid membrane lipids in MT

Previously, $\mathrm{He}$ et al. $^{4}$ reported that a decreased wax content at the mature stage results in the glossy surface of the mutant. Our research further demonstrates that wax biosynthesis in MT had already been inhibited at the fruit expansion stage, and the inhibition reached the highest level in the mature stage. Conversely, lipidomic data acquired from two independent years (2014 and 2017) 


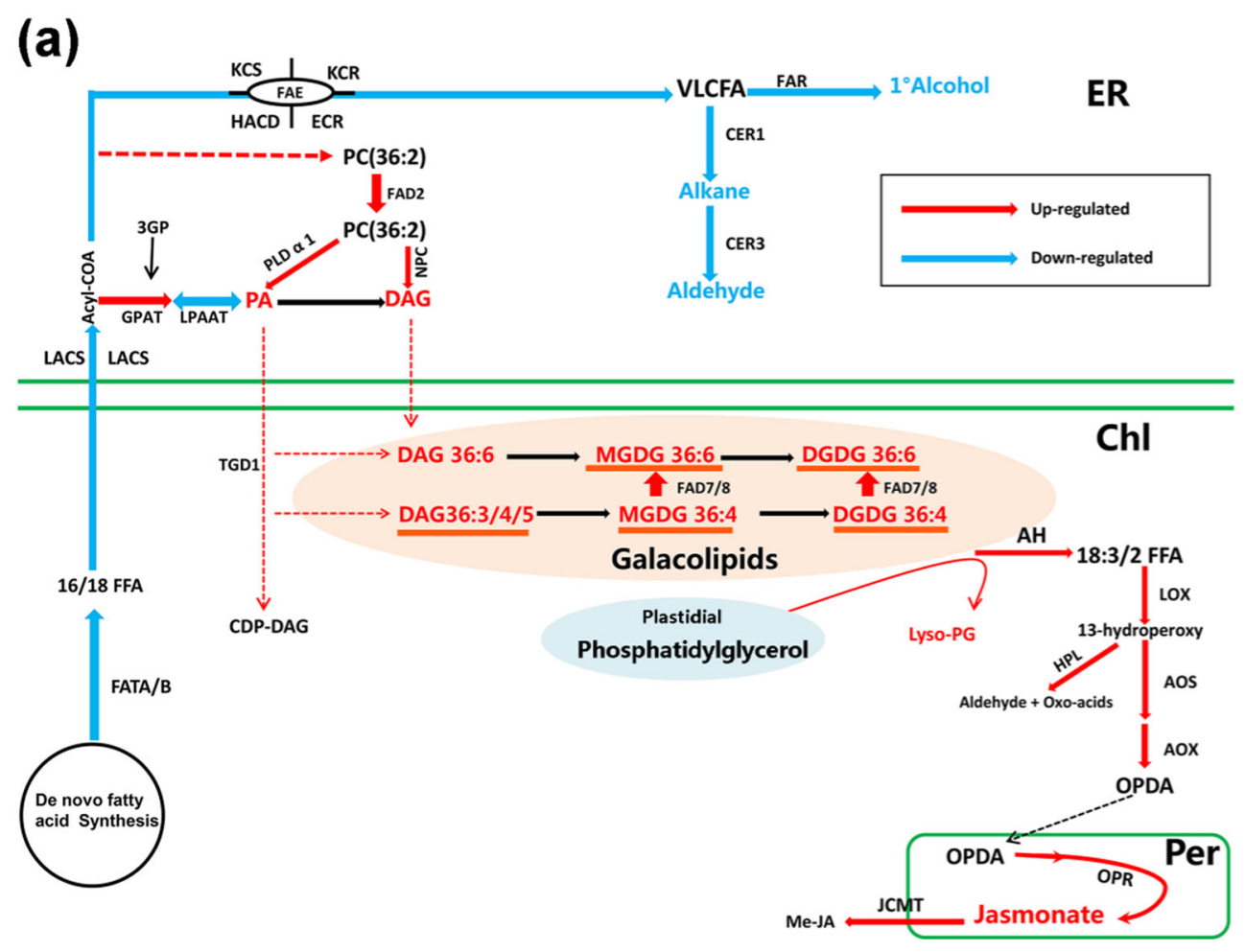

(b)
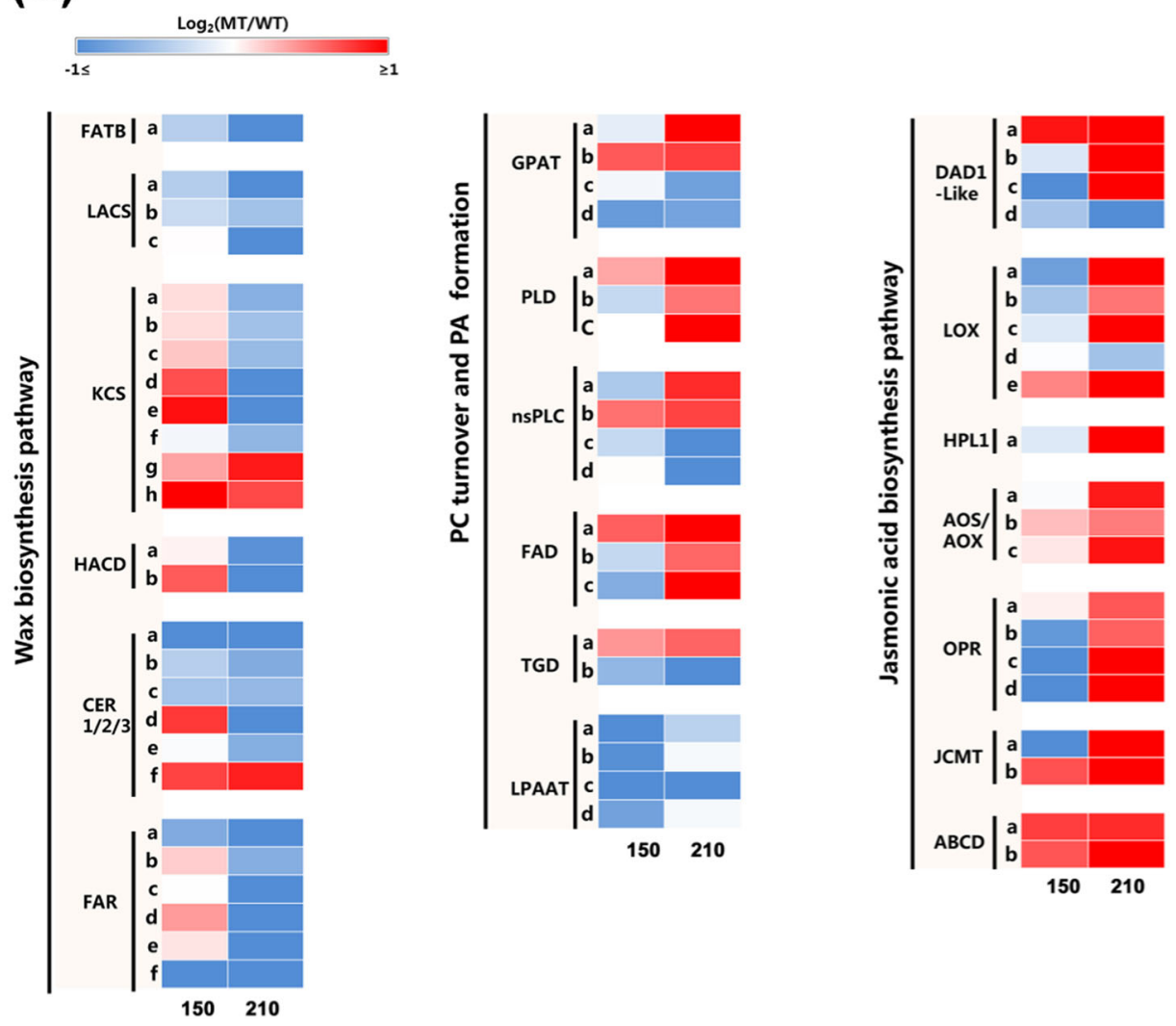

Fig. 6 (See legend on next page.) 
(see figure on previous page)

Fig. 6 Altered lipid pathways observed in MT at the mature stage. a Schematic diagram revealing the main altered lipid pathways at the transcriptional and metabolic levels in MT. The diagram was drawn based on data at the mature stage. Arrows in red or blue indicate transcriptionally up- or down-regulated metabolism pathways; lines in black denote that no genes were found based on our set of standards for transcriptome data; orange lines below phospholipids indicate positive correlation. Characters in red or blue represent up- or down-regulated metabolites. b Heatmaps showing $\log _{2}$ fold changes of important genes identified for wax, lipid and JA synthesis at the two stages, and the fold change was calculated as MT/ WT at the same time point. The genes were identified by a homologous BLAST search against known Arabidopsis genes (see "Materials and methods"). More detailed information is available in Supplementary Table S5. Numbers below indicate days after anthesis. ER endoplasmic reticulum, $\mathrm{Chl}$ chloroplast, Per peroxisome. FATB fatty acyl-ACP thioesterase B, LACS long-chain acyl-CoA synthetase, KCS ketoacyl-CoA synthase, HACD hydroxyacyl-CoA dehydratase, KCR ketoacyl-CoA reductase, ECR enoyl-CoA reductase, FAR fatty acyl-CoA reductase, CER 1/2/3 eceriferum 1/2/3, GPAT glycerol-3-phosphate acyltransferase, LPAAT 1-acylglycerol-3-phosphate acyltransferase, PLD phospholipase D, nsPLC nonspecific phospholipase C, FAD fatty acid desaturase, AH fatty acyl omega-hydroxylase, LOX lipoxygenase, HPL hydroperoxide lyase, AOS allene oxide synthase, AOX allene oxide cyclase, OPR oxo-phytodienoic acid reductase, JCMT jasmonic acid carboxyl methyltransferase, TGD trigalactosyl diacylglycerol, ABCD ATP-binding cassette acyl transporter D, PC phosphatidylcholine, PG phosphatidylglycerol, DAG diacylglycerol, PA phosphatidic acid, MGDG monogalactosyldiacylglycerol, DGDG digalactosyl diacylglycerol, VLCFA very-long-chain-fatty acid, CDP-DAG CDP-diacylglycerol, FA fatty acid, OPDA oxo-phytodienoic acid, Me-JA methyl jasmonate

demonstrated more dramatic increases in lipids in the mature stage than in the immature stage (Fig. 2b). The opposite accumulation trend of wax and plastid lipids in MT was also verified at the transcriptional level (Supplementary Table S5). These results suggest that the reduction in wax and the increase in chloroplast lipids occur simultaneously during fruit development. One possible reason for these alterations is the competition for FA substrates. FAs translocated from the plastid to the ER could either be used for the biosynthesis of various aliphatic wax components or be used for the biosynthesis of plastid-associated membranes ${ }^{36}$. The competition could be well-reflected by the divergent regulation profiles of KCS gene families, which function to generate VLCFA precursors for the formation of various lipids (Supplementary Table S5). Similar carbon alterations were observed in the tomato cuticular mutant named cutin deficient $2(c d 2)$. In this mutant, cuticular wax components, including alkanes and aldehydes, were reduced, and most galactolipids, together with some phospholipids, such as 34:3, 36:5 DAG, and 34:2 PG, were increased ${ }^{37}$. In addition, simultaneous alterations of cuticle compounds and plastid lipids were also found in many mutants, such as the mutants of acp3, acp 4 (acyl carrier protein 3, acyl carrier protein 4), acbp3 (acyl-CoA-binding protein 3), lacs2, lacs3, lacs4, and fad7, which showed simultaneous reduction of MGDG/DGDG lipids and wax components ${ }^{38-40}$. However, a tighter connection was observed between the biosynthesis of wax and eukaryotic plastid lipids. As reported in acbp3, only eukaryotic-derived DGDGs and not prokaryotic-derived DGDGs were significantly decreased together with severely suppressed wax biosynthesis ${ }^{39,41}$.

As mentioned above, more carbon flux into plastid lipids could have an influence on the energy capture ability of plants, as indicated by the significantly enriched photosynthesis pathways in KEGG (Fig. 5b). Moreover, the possibly altered photosynthesis may also be reflected by the upregulation of all four members of the pyruvate kinase (PK) gene family in MT, which are involved in the transformation of photosynthetic carbon precursors for FA synthesis. The four genes all showed a high correlation with plastid lipids (Table 1). Thus, the enhanced photosynthetic ability, together with the increase in unsaturated fatty acid species in MT, may greatly contribute to the resistance of MT to disease and stress. Such a complementary carbon flux between wax and chloroplast lipids may facilitate a more effective adaptation of the plant to the environment.

\section{Key factors affecting carbon distribution between wax and chloroplast lipids in MT}

Wax transporters may be involved in the altered carbon distribution between wax and plastid lipids, as we characterized two ABCG transporters, CsABCG15 and CsABCG32, which were downregulated in MT and negatively correlated with plastid lipids (Table 1). AtABCG15 shows the highest sequence similarity to the wax transporter CER5, and a 55\% reduction in wax levels was observed in the stem of the cer 5 mutant of Arabidopsis $^{42}$. CsABCG15 was found to be significantly downregulated in two glossy mutants of the "Newhall" navel orange, indicating a pivotal role of the gene in wax formation. Transcription factors may also play a role in the altered carbon distribution. WRI4 was reported to regulate wax formation through activation of LACS1, KCR, etc. ${ }^{43}$. Here, we found significant downregulation of WRI4 and LACS1 in MT, and both genes synergistically negatively correlated with plastid lipids. However, we did not find sequence differences of these genes between WT and MT; thus, these processes were unlikely to be the cause of the alteration. Lipid genes were also involved in this process. CsFAD2 showed upregulation in MT and a positive correlation with the two main 36:6 galactolipids, which demonstrated a clear activation of the eukaryotic pathway for galactolipid formation in MT. In addition, the 
(a)

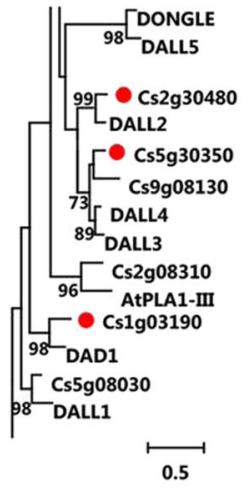

(c)

MGDG classes (35S:DAD1)

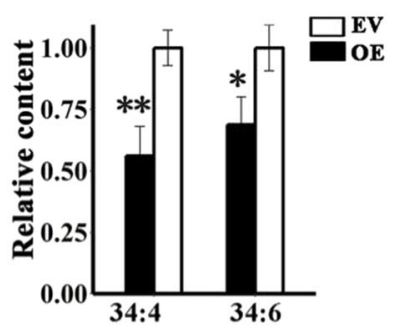

(f)

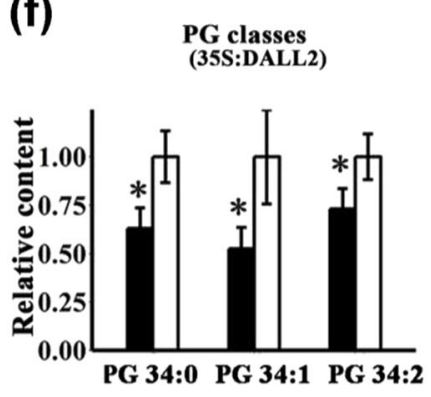

(b)
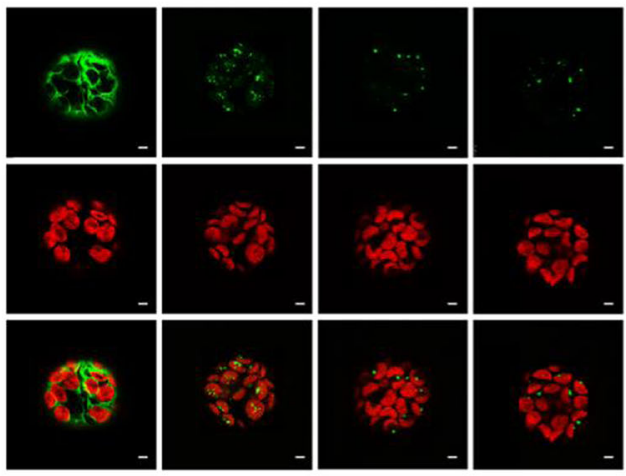

EV (d)

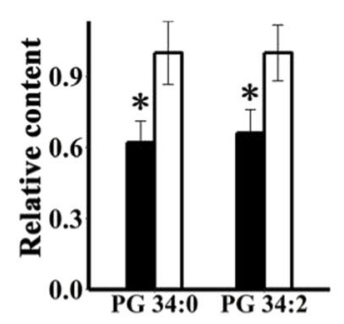

(g)

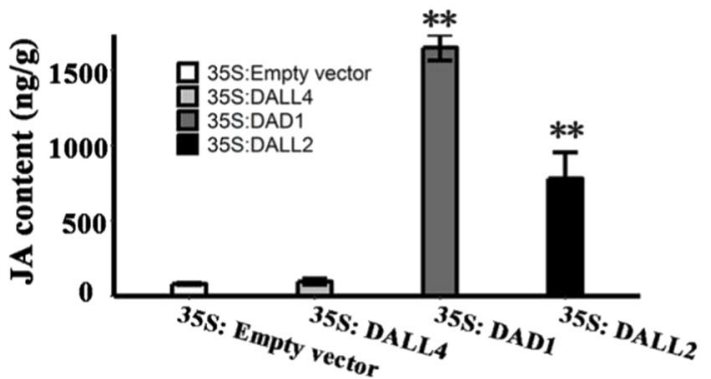

Fig. 7 Functional characterization of DAD1-like genes in $\mathbf{N}$. benthamiana. a Phylogenetic analysis of DAD1-like phospholipase genes in orange and their homologs in Arabidopsis. The numbers next to the branch represent the bootstrap scores (1000 replicates). The scale bar indicates the evolutionary distances computed using the JTT matrix-based method. More detailed information is available in Supplementary Fig. S7. b Subcellular localization of the three DAD1-like genes CsDALL4 (Cs5g30350), CsDAD1 (Cs1g03190), and CsDALL2 (Cs2g30480) in protoplasts of N. benthamiana. Green and red signals represent GFP and chlorophyll autofluorescence, respectively. Scale bar: $3 \mu \mathrm{m}$. c-f Relative contents of significantly changed phospholipid classes in overexpression lines of the three DAD1-like genes compared with EV. $\mathbf{c}$, $\mathbf{d}$ Relative contents of significantly changed MGDG and PG classes observed in overexpression lines of CsDAD1. e, f Relative contents of significantly changed MGDG and PG classes observed in overexpression lines of CSDALL2. Corrected areas of each lipid subclass in replicates were summed and normalized by the average number of EV controls. Bars are the average of three normalized biological replicates \pm standard deviation $(\mathrm{SD}, n=3)$. An asterisk $\left(^{*}\right)$ indicates a t-test $p$ value $\leq 0.05$ and double asterisks $\left(^{* *}\right)$ indicate a $t$-test $p$ value $\leq 0.01$. OE overexpression lines, EV empty vector, PG phosphatidylglycerol, MGDG

monogalactosyldiacylglycerol. $\mathbf{g}$ Content of JA in overexpression lines of the three DAD1-like genes and EV. Bars are the average of three biological replicates \pm standard deviation (SD, $n=3$ ). JA Jasmonic acid

expression level of FAD2 was found to be positively correlated with the eukaryotic pathway in suboptimal temperatures either in the "16:3 plant" Arabidopsis or in the "18:3 plant" wheat ${ }^{44}$. The increase in fully desaturated 36:6-carbon MGDG and DGDG at the mature stage in MT could have resulted from the increase in FAD7 expression. Mutants of fad7-1 and its closely related homolog $\mathrm{fad} 8$ exhibited decreased plastid lipid levels, which further resulted in low JA levels due to the lack of lipid precursors ${ }^{45,46}$.

FATB prefers saturated acyl-ACP as the substrate to release free FAs to the ER, and decreased wax crystals 
were found in fatb in Arabidopsis ${ }^{47}$. We also found that CsFATB was downregulated in two glossy mutants of the "Newhall" navel orange (Table 1). Interestingly, the gene also showed a highly negative correlation with the three main plastid lipids, which may be the result of elevated FA export from chloroplasts, as $40 \%$ and $55 \%$ increases in FA synthesis and oleate acid export were found in fatb, respectively, in an attempt to maintain lipid homeostasis $^{10}$. However, how these increased FAs were assimilated into PCs for eukaryotic plastid lipid biosynthesis remains unknown. The two upregulated GPATs (acyl-CoA: glycerol-3-phosphate acyltransferase) that putatively initiate the de novo synthesis of the PA-DAGPC pathway were expressed at much lower levels and thus may be insufficient for the biosynthesis of bulk amounts of PC. Further analysis showed that all members in the LPAAT (acyl-CoA: lysophosphatidic acid acyltransferase) gene family were downregulated, which may be explained by the fact that most lysophospholipid acyltransferases have forward and reverse functions or that the transcriptome variation may be inconsistent with the lipidomic data due to the involvement of some important posttranscriptional processes. Further studies are needed to clarify how fatty acids are used for the biosynthesis of PCs.

\section{Initiation of jasmonate synthesis in the "Newhall" navel orange}

Lipid degradation is indispensable in the maintenance of lipid homeostasis and lipid-mediated signaling ${ }^{48}$. Sequence comparison and phylogenetic analyses of PLA lipases identified in citrus and Arabidopsis showed that all three upregulated lipases were clustered into the DAD1like gene subgroup. The chloroplast subcellular location of the three genes suggested their roles as chloroplastlocalized lipases (Fig. 7b), which is consistent with previous reports in Arabidopsis that all DAD1-like gene members are located in the chloroplast ${ }^{32}$. We found that both plastid lipid classes, including galactolipids (MGDGs and DGDGs) and phospholipids (PGs), were significantly reduced in CsDAD1 and CsDALL2 overexpression lines, which is consistent with the results of in vitro studies that AtDAD1 and AtDALL2 could hydrolyze both phospholipids and galactolipids ${ }^{32,49}$. Furthermore, our results showed a 34-carbon substrate preference of CsDAD1, as indicated by the significant decrease in 34:0 and 34:2 PGs and highly unsaturated galacotolipids, such as $34: 4$ and 34:6 MGDGs (Fig. 7c, d). AtDALL2 showed the strongest activity toward MGDG in vitro ${ }^{32}$, and we did find that 34:4, 36:4, and 36:2 MGDGs were decreased in OE lines. Furthermore, we verified that this gene may also hydrolyze PGs, as indicated by the significant decrease in three PG subclasses in the OE lines (Fig. 7f). Consistent with the dramatic degradation of plastid lipids, we observed significantly elevated levels of JA in CsDAD1 and CsDALL2 overexpression lines, and in the CsDALL4 OE lines with few degraded lipids, we observed almost equal amounts of JA compared with that in the EV. Thus, the functional characterization results of the three genes were in accordance with our plastid-gene correlation prediction that CsDALL4 showed no high correlation with plastid lipids and possessed less plastid lipid hydrolyzation ability.

Based on the characterized function of the upregulated lipases in this study, we hypothesized that the elevated JA levels in MT may result from the enhanced lipid degradation pathways catalyzed by lipases to maintain chloroplast homeostasis, which is of great importance for the integrity of chloroplasts ${ }^{15}$. Given that CsDAD1 and CsDALL2 both presented little preference for 36:6 MGDG and DGDG, which account for over $60 \%$ of the respective lipid classes and displayed elevated levels in MT (Supplementary Table S8), there should be other chloroplast-localized lipases involved in the process. These lipases could be characterized in future work to fully identify the key lipase genes that initiate JA synthesis in "Newhall" navel oranges.

\section{Materials and methods}

Fruits of the mutant (MT) and wild type (WT) were harvested in three adjacent orchards (one contained mutant trees, and the other two contained the grafted branches from the MT) in Anyuan County of Jiangxi Province in China in 2014 and 2017 with similar management conditions. Three biological replicates of MT and WT samples were collected in the preharvest stage at 90 DAA, 150 DAA, and 210 DAA, with each replicate containing at least ten representative fruits of uniform size. Flavedo tissues were rapidly sampled from each fruit, immediately immersed in liquid nitrogen, and then stored at $-80^{\circ} \mathrm{C}$ for transcriptomic and metabolomic analysis.

\section{Total wax extraction and analysis by GC-MS}

The total wax was extracted according to a previously reported method ${ }^{4,23}$. Intact fruits were immersed in chloroform for $2 \mathrm{~min}$, twice. After the addition of $200 \mu \mathrm{l}$ $(1 \mu \mathrm{g} / \mu \mathrm{l}) \mathrm{n}$-tetracosane as an internal standard, the extracts were dried by a gentle stream of nitrogen $\left(\mathrm{N}_{2}\right)$ and then derivatized in pyridine for $30 \mathrm{~min}$ at $50^{\circ} \mathrm{C}$, followed by treatment with bis-N,N-(trimethylsilyl) trifluoroacetamide (BSTFA) containing 1\% trimethylchlorosilane (Sigma) for $40 \mathrm{~min}$ at $60^{\circ} \mathrm{C}$. After derivatization, the mixture was concentrated with a gentle nitrogen gas flow and redissolved by chloroform. GC-MS (Thermo Fisher, ISQII, USA) and an Agilent DB-1 column $(30 \mathrm{~m} \times 25 \mu \mathrm{m}$ i.d. $\times 0.1 \mu \mathrm{m})$ were used to perform wax analysis. The instrument parameter settings were consistent with those of previous reports ${ }^{23}$. The contents of all acquired compounds were calculated by comparison 
with the internal standard. Three biological replicates at 90 DAA, 150 DAA, and 210 DAA were analyzed.

\section{Lipid extraction and analysis using UPLC-MS/MS}

Lipids were extracted from fruits of the WT and MT collected at $150 \mathrm{DAA}$ and $210 \mathrm{DAA}$ using the protocol described below: Briefly, $30 \mathrm{mg}$ of freeze-dried flavedo tissue was ground into a fine powder. Metabolites from each aliquot were extracted with $1 \mathrm{ml}$ of precooled $\left(-20^{\circ} \mathrm{C}\right)$ extraction buffer (homogenous methanol/ methyl-tert-butyl-ether [1:3] mixture). After $10 \mathrm{~min}$ of incubation at $4{ }^{\circ} \mathrm{C}$ and sonication for $10 \mathrm{~min}$ in an ultrasonication bath, $500 \mu \mathrm{l}$ of a water/methanol mixture was added. Samples were then centrifuged ( $5 \mathrm{~min}, 20,817 \mathrm{~g}$ ), which led to the formation of two phases: a lipophilic phase and a polar phase. A lipophilic phase of $500 \mathrm{ml}$ was collected and dried under vacuum. The lipophilic phase was resuspended in $200 \mu \mathrm{l}$ of isopropanol and used for lipid analysis.

Lipid analysis was performed on a 5600 plus AccurateMass Q-TOF (AB SCIEX 5600 plus Q-TOF, USA) mass spectrometer system. Liquid chromatography was carried out using a UPLC with an autosampler (Shimadzu Corporation, Kyoto, Japan). An ODS C-18 (Shimadzu Corporation) column $(2.1 \mathrm{~mm} \times 150 \mathrm{~mm}, 2 \mu \mathrm{m})$ was used at $40^{\circ} \mathrm{C}$. The injected volume of the sample was $10 \mu \mathrm{L}$. The elution gradient was carried out with a binary solvent system consisting of $10 \mathrm{mM}$ ammonium acetate in 30\% methanol in water (solvent $\mathrm{A}$ ) and $10 \mathrm{mM}$ ammonium acetate in $10 \%$ acetonitrile in isopropanol (solvent $\mathrm{B}$ ) at a constant flow rate of $250 \mu \mathrm{l} / \mathrm{min}$. A linear gradient profile with the following proportions $(\mathrm{v} / \mathrm{v})$ of solvent $\mathrm{B}$ was applied: 0-2 $\mathrm{min}, 25 \% \mathrm{~B} ; 2-5 \mathrm{~min}, 25-40 \% \mathrm{~B} ; 4-22 \mathrm{~min}$, $40-95 \%$ B, 22-27 min, 95\% B; 27-27.5 min, $100-25 \%$ B; and $27.5-32 \mathrm{~min}, 4.5 \mathrm{~min}$ for re-equilibration at $25 \% \mathrm{~B}$. The MS spectra were processed with Analyst TF 1.7 (SCIEX, USA). MS conditions were as follows: source voltage, $5.5 \mathrm{kV}$; source temperature, $550^{\circ} \mathrm{C}$; declustering potential, $100 \mathrm{~V}$; gas 1 and 2 (nitrogen), $50 \mathrm{psi}$; and curtain gas (nitrogen), 35 psi. A data-dependent analysis was conducted on the 15 most abundant metabolite ions in a full-scan cycle $(100 \mathrm{~ms})$. The scan ranges of $\mathrm{m} / \mathrm{z}$ of precursor ion and product ion were set as $400-1000 \mathrm{Da}$ and 80-1000 Da, respectively. The selected precursor ions were $>4000$ cps with a charge state of 1 at an MS tolerance of $50 \mathrm{mDa}$. The collision energy of the fragment in the collision cell was set at $30 \pm 10 \mathrm{eV}$. Compounds were identified based on their accurate mass for which tolerance was set to a $5 \mathrm{ppm}$ mass window and the MS2 fragmentation pattern of the precursor ion. The data were obtained using Analyst TF 1.6 and MultiQuantTM and processed using PeakView 2.0 software. Accurate molecular weight and fragmentation ion information were acquired using LipidView 2.0 to generate an in-house dataset. The identification of glycerolipid classes and species was based on retention time, accurate $m / z$, and fragmentation ion patterns according to a previous report $^{50}$.

\section{Measurement of JA phytohormones in "Newhall" navel orange and $\mathrm{N}$. benthamiana}

Measurement of JA was performed according to a previously reported method ( $\mathrm{He}$ et $\mathrm{al}^{4}{ }^{4}$ ). One hundred milligrams of each samples was ground and mixed with $800 \mu \mathrm{l}$ of solvent containing methanol $/ \mathrm{H}_{2} \mathrm{O} /$ acetic acid (80:19:1, v/v/v) and internal standards for JA determination (DHJA; Olomouc, Czech Republic). The analysis of hormone extracts was performed using an Agilent 1100 HPLC system coupled to an Agilent API3000 mass spectrometer. Identification and annotation of JA were conducted according to a reported method ${ }^{51}$. Three biological replicates of "Newhall" navel oranges (WT and $\mathrm{MT}$ ) and $N$. benthamiana (overexpression lines and control lines) were utilized. Flavedo and juice sac tissues in "Newhall" navel oranges and infiltrated leaves of $N$. benthamiana were used.

\section{Flavedo RNA-seq analysis and qRT-PCR validation}

Flavedo tissues sampled from WT and MT at 150 DAA and 210 DAA were used for RNA extraction with the method reported by Liu et al. ${ }^{52}$. Stranded mRNA-Seq libraries were constructed using the KAPA Stranded mRNA-seq kit (Kapa Biosystems, Cape Town, SA) following the manufacturer's recommendations and were sequenced with the Illumina HiSeq X Ten system (pairedend 150-bp reads) with three biological replicates for 150 DAA, two for 210 DAA in 2014 and three for 210 DAA in 2017. Approximately eight million clean reads were mapped to the reference genome from Citrus sinensis ${ }^{53}$ using Tophat (with the G parameter). The uniquely mapped reads were extracted for estimating the expression levels of protein-coding genes using Cufflinks, and FPKM was used as the unit of measurement to estimate transcript abundance. Annotation of genes was based on the data acquired from the $C$. sinensis annotation website: http://citrus.hzau.edu.cn/orange/index.php. For DEG analysis, a count table was first generated by featureCounts. DEGs were identified by applying statistical tests to the WT and MT groups in the same stage, which was performed using DESeq2 in the R statistical package. All genes with a Benjamini-Hochberg adjusted $p$ value smaller than 0.05 and with a FC larger than 1.5 were reported as DEGs.

A total of 15 genes putatively involved in lipid and wax pathways were used to validate the RNA-seq results through quantitative real-time polymerase chain reaction (qRT-PCR), and the results are presented in Supplementary Figs. S9 and S10. Real-time qRT-PCR was performed 
on the Roche LightCycler 480II System (Roche Diagnostics). The acquired data were processed with the $2^{-\Delta \Delta \mathrm{Ct}}$ analysis method ${ }^{54}$.

Protein sequences of DEGs were extracted and submitted to KOBAS3.0 for KEGG enrichment analysis ${ }^{55}$. Protein sequences of genes that showed a high positive correlation with the three plastid lipids were extracted and submitted to the PlantTFDB 4.0 database (http:// planttfdb.cbi.pku.edu.cn/index.php) for GO enrichment analysis.

\section{Analysis of lipid-related genes in the "Newhall" navel orange and annotation of transcription factors}

To identify lipid-related genes in the "Newhall" navel orange, the protein sequences of lipid-related genes in Arabidopsis were used to run the BLASTp program (Blast + version 2.3.0) against protein sequences of all up/ downregulated genes in the "Newhall" navel orange, with a threshold of an $E$-value $<\mathrm{e}^{-6}$ and an identity $\geq 30 \%$. The extracted sequences were further confirmed with conserved domains using HMMER (v3.1b2) (http://hmmer. org/). The results are shown in Supplementary Table S5.

\section{Phylogenetic analysis}

Multiple sequence alignment was performed using the MUSCLE program based on amino acid sequences of phospholipase A family genes in Arabidopsis and Citrus and then manually adjusted in MEGA (version 7.0.14). The maximum likelihood phylogenetic tree was constructed using MEGA (version 7.0.14) with the JTT model and 1000 bootstrap resamplings. Bootstrap values above 70 are shown. Gene IDs used to generate phylogenetic trees are provided in Supplementary Table S7, and the results of the phylogenetic tree are shown in Supplementary Fig. S7.

\section{Subcellular localization of CsDAD1, CsDALL2, and CsDALL4}

The 35S:CsDAD1-GFP, 35S:CsDALL2-GFP, and 35S: CsDALL4-GFP fusion constructs were produced by inserting the coding sequence without a stop codon into pH7LIC6.0 (35S-GFP). The GFP fusion constructs were introduced into protoplasts prepared from $N$. benthamiana rosette leaves by polyethylene glycol-mediated transformation ${ }^{56}$. Protoplasts were incubated at $24^{\circ} \mathrm{C}$ in the dark for $18 \mathrm{~h}$. The florescence images were captured using a confocal laser-scanning microscope (TCS SP2; Leica, Wetzlar, Germany). GFP signals were imaged with excitation at $488 \mathrm{~nm}$, and the emission signal was collected between 500 and $555 \mathrm{~nm}$. The autofluorescence signal of chlorophyll was collected between 650 and $750 \mathrm{~nm}$. Details of primers for constructs are listed in Supplementary Table S9.

\section{Transient overexpression of CsDAD1, CsDALL2, and CsDALL4 in N. benthamiana}

Transient expression in $N$. benthamiana leaves was performed as previously reported ${ }^{57,58}$ with some minor modifications. The full-length cDNA of the three genes was amplified and cloned into the Gateway Entry vector pDNOR 221, which was then recombined with the resulting plasmids PK7WG2D. Two Agrobacterium tumefaciens GV3101 strains harboring either the constructed vector or the gene coding for the p19 viral suppressor protein were mixed together so that the final OD 600 of each culture was equal to 0.2 prior to infiltration. Infiltration was performed on the underside of leaves of 1month-old $N$. benthamiana that had been cultured in a $24{ }^{\circ} \mathrm{C}$ plant growth room with a $10: 14$ dark:light cycle. Infiltration experiments were conducted in triplicate, and each replicate contained nine leaves harvested from three plants with uniform size. After infiltration, $N$. benthamiana plants were grown for another 5 days before leaf discs were harvested, pooled and stored at $-80^{\circ} \mathrm{C}$. The method used for $N$. benthamiana lipid analysis was the same as described above. The primers used are listed in Supplementary Table S9.

\section{Acknowledgements \\ This research was financed by funding from the National Natural Science Foundation of China (31772261) and the Huazhong Agricultural University Scientific \& Technological Self-Innovation Foundation granted to W.W. We would like to thank Prof. Zuoxiong Liu from the Foreign Language School of Huazhong Agricultural University for revising and improving the English language of the paper. We would also like thank Prof. Feng Li (Huazhong Agricultural University) for the provision of all vectors used in this study. In addition, we would like to thank Prof. Liang Guo and Shaoping Lu for valuable discussions.}

\section{Author details \\ ${ }^{1}$ Key Laboratory of Horticultural Plant Biology (MOE), College of Horticulture and Forestry Sciences, Huazhong Agricultural University, 430070 Wuhan, China. ${ }^{2}$ National Key Laboratory of Crop Genetic Improvement, Huazhong Agricultural University, 430070 Wuhan, China. ${ }^{3}$ Key Laboratory for Space Bioscience and Biotechnology, School of Life Sciences, Northwestern Polytechnical University, Youyi Xilu 127, Xi'an 710072 Shaanxi, China. ${ }^{4}$ Department of Life Sciences, Ben-Gurion University of the Negev, Beersheba, Israel. ${ }^{5}$ Max-Planck-Institute of Molecular Plant Physiology, Am Muehlenberg 1, 14476 Potsdam, Germany}

\section{Conflict of interest}

The authors declare that they have no conflict of interest.

Supplementary Information accompanies this paper at (https://doi.org/ 10.1038/s41438-020-0262-z).

Received: 26 July 2019 Revised: 26 December 2019 Accepted: 7 February 2020

Published online: 01 April 2020

\footnotetext{
References

1. Ben-Yehoshua, S., Burg, S. P. \& Young, R. Resistance of citrus fruit to mass transport of water vapor and other gases. Plant Physiol. 79, 1048-1053 (1985).
} 
2. El-Otmani, M., Coggins Jr, C. \& Eaks, I. Fruit age and gibberellic acid effect on epicuticular wax accumulation, respiration, and internal atmosphere of nave orange fruit. J. Am. Soc. Hortic. Sci. 111, 228-232 (1986).

3. Liu, D. C. et al. A comparison of the ultrastructure and composition of fruits cuticular wax from the wild-type 'Newhall' navel orange (Citrus sinensis [L.] Osbeck cv. Newhall) and its glossy mutant. Plant Cell Rep. 31, 2239-2246 (2012).

4. He, Y. et al. Integrated transcriptomic and metabolomic analyses of a wax deficient citrus mutant exhibiting jasmonic acid-mediated defense against fungal pathogens. Hortic. Res. 5, 43 (2018).

5. Hadley, N. F. Lipid water barriers in biological systems. Prog. Lipid Res. 28, 1-33 (1989).

6. Boudiere, L. et al. Glycerolipids in photosynthesis: composition, synthesis and trafficking. Biochim. Biophys. Acta 1837, 470-480 (2014).

7. Hurlock, A. K., Roston, R. L., Wang, K. \& Benning, C. Lipid trafficking in plant cells. Traffic 15, 915-932 (2014).

8. Voisin, D. et al. Dissection of the complex phenotype in cuticular mutants of Arabidopsis reveals a role of SERRATE as a mediator. PLoS Genet. 5, e1000703 (2009).

9. Kunst, L. \& Samuels, A. L. Biosynthesis and secretion of plant cuticular wax. Prog. Lipid Res. 42, 51-80 (2003).

10. Gustavo, B., Xiaoming, B., John, O. \& Mike, P. Metabolic responses to the reduction in palmitate caused by disruption of the FATB gene in Arabidopsis. Plant Physiol. 135, 1269-1279 (2004).

11. Jones, M. R. Lipids in photosynthetic reaction centres: Structural roles and functional holes. Prog. Lipid Res. 46, 56-87 (2007).

12. Fujii, S., Kobayashi, K., Nagata, N., Masuda, T. \& Wada, H. Monogalactosyldiacylglycerol facilitates synthesis of photoactive protochlorophyllide in etioplasts. Plant Physiol. 174, 2183-2198 (2017).

13. Zhang, M. et al. Modulated fatty acid desaturation via overexpression of two distinct omega-3 desaturases differentially alters tolerance to various abiotic stresses in transgenic tobacco cells and plants. Plant J. 44, 361-371 (2010).

14. Liu, X. Y. et al. Overexpression of tomato chloroplast omega-3 fatty acid desaturase gene alleviates the photoinhibition of photosystems 2 and 1 under chilling stress. Photosynthetica 46, 185-192 (2008).

15. Moellering, E. R. \& Benning, C. Galactoglycerolipid metabolism under stress: a time for remodeling. Trends Plant Sci. 16, 98-107 (2011).

16. Ryu, S. B. Phospholipid-derived signaling mediated by phospholipase A in plants. Trends Plant Sci. 9, 229-235 (2004).

17. Richmond, G. S. \& Smith, T. K. Phospholipases, A1. Int. J. Mol. Sci. 12, 588-612 (2011).

18. Ellinger, D. et al. DONGLE and DEFECTIVE IN ANTHER DEHISCENCE1 lipases are not essential for wound- and pathogen-induced jasmonate biosynthesis: redundant lipases contribute to jasmonate formation. Plant Physiol. 153 114-127 (2010).

19. Wasternack, C. H. B. Jasmonates: biosynthesis, perception, signal transduction and action in plant stress response, growth and development. An update to the 2007 review in Annals of Botany. Ann. Bot. 111, 1021-1058 (2013).

20. Wang, K. et al. Two abscisic acid-responsive plastid lipase genes involved in jasmonic acid biosynthesis in Arabidopsis thaliana. Plant Cell 30, 1006-1022 (2018).

21. Christeller, J. T. \& Galis, I. alpha-linolenic acid concentration and not wounding per se is the key regulator of octadecanoid (oxylipin) pathway activity in rice (Oryza sativa L.) leaves. Plant Physiol. Biochem. 83, 117-125 (2014).

22. Wang, J. et al. Regulation of cuticle formation during fruit development and ripening in 'Newhall' navel orange (Citrus sinensis Osbeck) revealed by transcriptomic and metabolomic profiling. Plant Sci.: Int. J. Exp. Plant Biol. 243 131-144 (2016).

23. Wang, J. et al. Comparative analysis of surface wax in mature fruits between Satsuma mandarin (Citrus unshiu) and 'Newhall' navel orange (Citrus sinensis) from the perspective of crystal morphology, chemical composition and key gene expression. Food Chem. 153, 177-185 (2014).

24. Li-Beisson, Y. et al. Acyl-lipid metabolism. Arabidopsis Book 11, e0161. https:// doi.org/10.1199/tab.016110.1199/tab.0133 (2013).

25. Liu, D. et al. Transcriptome sequencing identified wax-related genes controlling the glossy phenotype formation of "Ganqi 3," a bud mutant derived from wild-type "Newhall" navel orange. Tree Genet. Genom. 12, https:/doi.org/ 10.1007/s11295-016-1017-8 (2016)

26. Ohlrogge, J. \& Browse, J. Lipid biosynthesis. Plant Cell 7, 957 (1995).
27. Fabre, G. et al. The ABCG transporter PEC1/ABCG32 is required for the formation of the developing leaf cuticle in Arabidopsis. New Phytologist 209, 192-201 (2016).

28. Li, L. et al. The maize glossy13 gene, cloned via BSR-Seq and Seq-walking encodes a putative $A B C$ transporter required for the normal accumulation of epicuticular waxes. PLoS ONE 8, e82333 (2013).

29. David, P. et al. The Arabidopsis DESPERADO/AtWBC11 transporter is required for cutin and wax secretion. Plant Physiol. 145, 1345-1360 (2007).

30. Xu, C., Fan, J., Froehlich, J. E., Awai, K. \& Benning, C. Mutation of the TGD1 chloroplast envelope protein affects phosphatidate metabolism in Arabidopsis. Plant Cell 17, 3094-3110 (2005).

31. Gimenez-lbanez, S. et al. JAZ2 controls stomata dynamics during bacterial invasion. New Phytologist 213, 1378-1392 (2017)

32. Seo, Y. S., Kim, E. Y., Kim, J. H. \& Kim, W. T. Enzymatic characterization of class I DAD1-like acylhydrolase members targeted to chloroplast in Arabidopsis. FEBS Lett. 583, 2301-2307 (2009).

33. Wood, C. C. et al. A leaf-based assay using interchangeable design principles to rapidly assemble multistep recombinant pathways. Plant Biotechnol. J. 7, 914-924 (2009).

34. Dussert, S. et al. Comparative transcriptome analysis of three oil palm fruit and seed tissues that differ in oil content and fatty acid composition. Plant Physiol. 162, 1337-1358 (2013).

35. Yang, D.-H., Hettenhausen, C., Baldwin, I. T. \& Wu, J. Silencing Nicotiana attenuata calcium-dependent protein kinases, CDPK4 and CDPK5, strongly upregulates wound-and herbivory-induced jasmonic acid accumulations. Plant Physiol. 159, 1591-1607 (2012).

36. Jetter, R. \& Kunst, L. Plant surface lipid biosynthetic pathways and their utility for metabolic engineering of waxes and hydrocarbon biofuels. Plant J. 54, 670-683 (2008).

37. Kimbara, J. et al. Inhibition of CUTIN DEFICIENT 2 causes defects in cuticle function and structure and metabolite changes in tomato fruit. Plant Cell Physiol. 54, 1535-1548 (2013).

38. Xia, Y. et al. The glabra1 mutation affects cuticle formation and plant responses to microbes. Plant Physiol. 154, 833-846 (2010).

39. Xia, Y. et al. An intact cuticle in distal tissues is essential for the induction of systemic acquired resistance in plants. Cell Host Microbe 5, 151-165 (2009).

40. Gao, Q.-M. Glycerolipids and the Plant Cuticle Contribute to Plant Immunity. Theses and Dissertations-Plant Pathology. 4, https://uknowledge.uky.edu/ plantpath_etds/4 (2012).

41. Xia, Y. The Role of Cuticle, Fatty Acids, and Lipid Signaling in Plant Defense. Theses and Dissertations-Plant Pathology. 10, https://uknowledge.uky.edu/ plantpath_etds/10 (2010).

42. Pighin, J. A. et al. Plant cuticular lipid export requires an $A B C$ transporter Science 306, 702 (2004)

43. Park, C. S., Go, Y. S. \& Suh, M. C. Cuticular wax biosynthesis is positively regulated by WRINKLED 4, an AP 2/ERF-type transcription factor, in Arabidopsis stems. Plant J. 88, 257-270 (2016).

44. Li, Q. et al. Understanding the biochemical basis of temperature-induced lipid pathway adjustments in plants. Plant Cell 27, 86-103 (2015).

45. Hugly, S., Kunst, L. \& Somerville, C. Enhanced thermal tolerance of photosynthesis and altered chloroplast ultrastructure in a mutant of Arabidopsis deficient in lipid desaturation. Plant Physiol. 90, 1134-1142 (1989).

46. Stintzi, A., Weber, H., Reymond, P. \& Farmer, E. E. Plant defense in the absence of jasmonic acid: the role of cyclopentenones. Proc. Natl Acad. Sci. USA 98, 12837-12842 (2001)

47. Bonaventure, G., Salas, J. J., Pollard, M. R. \& Ohlrogge, J. B. Disruption of the FATB gene in Arabidopsis demonstrates an essential role of saturated fatty acids in plant growth. Plant Cell 15, 1020 (2003).

48. Lim, G. H., Singhal, R., Kachroo, A. \& Kachroo, P. Fatty acid- and lipid-mediated signaling in plant defense. Annu. Rev. Phytopathol. 55, 505 (2017).

49. Hyun, Y. et al. Cooperation and functional diversification of two closely related galactolipase genes for jasmonate biosynthesis. Dev. Cell 14, 183-192 (2008).

50. Lu, S., Liu, H., Jin, C., Li, Q. \& Guo, L. An efficient and comprehensive plant glycerolipids analysis approach based on high-performance liquid chromatography-quadrupole time-of-flight mass spectrometer. Plant Direct 3, e00183 (2019).

51. Liu, H., Li, X., Xiao, J. \& Wang, S. A convenient method for simultaneous quantification of multiple phytohormones and metabolites: application in study of rice-bacterium interaction. Plant Methods 8, 2 (2012). 
52. Liu, Y. Z., Liu, Q. \& Tao, N. G. Efficient isolation of RNA from fruit peel and pulp of ripening navel orange (Citrus sinensis Osbeck). J. Huazhong Agric. Univ. 25, 300-304 (2006).

53. $\mathrm{Xu}, \mathrm{Q}$. et al. The draft genome of sweet orange (Citrus sinensis). Nat. Genet. $\mathbf{4 5}$ 59-66 (2013).

54. Bustin, S. A. et al. The MIQE guidelines: minimum information for publication of quantitative real-time PCR experiments. Clin. Chem. 55, 611-622 (2009).

55. Xie, C. et al. KOBAS 2.0: a web server for annotation and identification of enriched pathways and diseases. Nucleic Acids Res. 39, W316-W322 (2011).
56. Yoo, S.-D., Cho, Y.-H. \& Sheen, J. Arabidopsis mesophyll protoplasts: a versatile cell system for transient gene expression analysis. Nat. Protoc. $\mathbf{2}$ 1565 (2007).

57. Olivier, V., Susana, R., Pere, M. \& David, B. An enhanced transient expression system in plants based on suppression of gene silencing by the p19 protein of tomato bushy stunt virus. Plant J. 33, 949-956 (2010).

58. Sparkes, I. A., Runions, J., Kearns, A. \& Hawes, C. Rapid, transient expression of fluorescent fusion proteins in tobacco plants and generation of stably transformed plants. Nat. Protoc. 1, 2019-2025 (2006). 